\title{
Short-Term Depression, Temporal Summation, and Onset Inhibition Shape Interval Tuning in Midbrain Neurons
}

\author{
Christa A. Baker and Bruce A. Carlson \\ Department of Biology, Washington University in St. Louis, St. Louis, Missouri 63130-4899
}

\begin{abstract}
A variety of synaptic mechanisms can contribute to single-neuron selectivity for temporal intervals in sensory stimuli. However, it remains unknown how these mechanisms interact to establish single-neuron sensitivity to temporal patterns of sensory stimulation in vivo. Here we address this question in a circuit that allows us to control the precise temporal patterns of synaptic input to interval-tuned neurons in behaviorally relevant ways. We obtained in vivo intracellular recordings under multiple levels of current clamp from midbrain neurons in the mormyrid weakly electric fish Brienomyrus brachyistius during stimulation with electrosensory pulse trains. To reveal the excitatory and inhibitory inputs onto interval-tuned neurons, we then estimated the synaptic conductances underlying responses. We found short-term depression in excitatory and inhibitory pathways onto all interval-tuned neurons. Short-interval selectivity was associated with excitation that depressed less than inhibition at short intervals, as well as temporally summating excitation. Long-interval selectivity was associated with long-lasting onset inhibition. We investigated tuning after separately nullifying the contributions of temporal summation and depression, and found the greatest diversity of interval selectivity among neurons when both mechanisms were at play. Furthermore, eliminating the effects of depression decreased sensitivity to directional changes in interval. These findings demonstrate that variation in depression and summation of excitation and inhibition helps to establish tuning to behaviorally relevant intervals in communication signals, and that depression contributes to neural coding of interval sequences. This work reveals for the first time how the interplay between short-term plasticity and temporal summation mediates the decoding of temporal sequences in awake, behaving animals.
\end{abstract}

Key words: electrosensory system; short-term synaptic plasticity; temporal coding; weakly electric fish

\section{Introduction}

Many circuits contain neurons tuned to particular timing sequences in sensory stimuli (Rose and Capranica, 1983; Grothe, 1994; Fortune and Rose, 1997a; Edwards et al., 2002; Sakai et al., 2009; Pluta and Kawasaki, 2010; Goel and Buonomano, 2014). Single-neuron temporal tuning reflects a combination of presynaptic tuning, synaptic filtering, and postsynaptic filtering. Two synaptic mechanisms that have been implicated in establishing temporal filtering in vivo are short-term depression (Fortune and Rose, 2000) and temporal summation (Rose et al., 2011). However, it is difficult to precisely determine the relative contributions of various synaptic filtering mechanisms to interval tuning in vivo because temporal filtering by presynaptic neurons makes the relationship between stimulus intervals and presynaptic input patterns unknown. In vitro slice recordings, which allow precise control of presynaptic stimulation, have also revealed that short-term plasticity (Klyachko and Stevens, 2006) and temporal

\footnotetext{
Received June 5, 2014; revised Aug. 20, 2014; accepted Sept. 7, 2014.

Author contributions: C.A.B. and B.A.C. designed research; C.A.B. performed research; C.A.B. analyzed data; C.A.B. and B.A.C. wrote the paper.

This work was supported by the National Science Foundation (Grant IOS-1050701 to B.A.C.) and the National Institute on Deafness and Other Communication Disorders (Grant F31-DC012452 to C.A.B.).

The authors declare no competing financial interests.

Correspondence should be addressed to Bruce A. Carlson, Washington University in St. Louis, Department of Biology, Campus Box 1137, One Brookings Drive, St. Louis, M0 63130-4899. E-mail: carlson.bruce@wustl.edu.

DOI:10.1523/JNEUROSCI.2299-14.2014

Copyright $\odot 2014$ the authors $\quad 0270-6474 / 14 / 3414272-16 \$ 15.00 / 0$
}

summation (George et al., 2011) act as temporal filters of synaptic input. However, in vitro preparations sever connections between neurons, thereby disrupting the natural spatiotemporal pattern of inputs. Furthermore, pharmacologically blocking inhibition may release presynaptic excitatory inputs from their natural inhibition, making it difficult to isolate the relative contributions of excitation and inhibition to single-neuron temporal selectivity under natural conditions.

Here we study the interactions of excitation and inhibition in interval-tuned neurons in vivo in a circuit that allows precise control of presynaptic timing. Mormyrid electric fish are ideal for studying synaptic mechanisms of temporal filtering because the interspike intervals of presynaptic inputs onto interval-tuned neurons precisely follow the interpulse intervals (IPIs) in sensory stimuli. Moreover, these intervals can be varied in ways that fish would naturally experience. Mormyrids vary the intervals between successive electric organ discharges (EODs) to communicate (for review, see Carlson, 2002). Natural IPIs range from $\sim 10$ ms to hundreds of milliseconds (Hopkins, 1986), and the ability to accurately detect and decode rapidly changing temporal patterns of EODs is essential for social interactions (Carlson, 2002).

Electric communication signals are processed by a dedicated sensory pathway (Xu-Friedman and Hopkins, 1999; Baker et al., 2013). Electroreceptors in the skin fire one time-locked spike in response to each EOD (Bennett, 1965). This spike pattern is relayed to the hindbrain nucleus of the electrosensory lateral line 
lobe, which in turn projects to the anterior exterolateral nucleus (ELa) of the midbrain torus semicircularis. The ELa sends its only output to the adjacent posterior EL (ELp) while preserving the IPIs of the sensory stimulus (Carlson, 2009). ELp neurons respond selectively to particular IPIs and thus act as temporal filters of afferent spike patterns (Carlson, 2009).

In the current study, we present electrosensory stimulation to awake, behaving fish, record responses of single ELp neurons to IPI trains under multiple levels of current clamp, and estimate the excitatory and inhibitory synaptic conductances underlying IPI tuning. This method maintains the intact neural circuit and allows direct comparison of the strength and time course of excitation and inhibition to study the relative contributions of short-term synaptic plasticity and temporal summation in establishing interval selectivity.

\section{Materials and Methods}

Animals. We used a total of 45 Brienomyrus brachyistius $(5.0-9.0 \mathrm{~cm}$ in standard length) of both sexes in this study. We acquired the fish through the aquarium trade and housed them in groups with a $12 \mathrm{~h}$ light/dark cycle, water conductivity of $200-400 \mu \mathrm{S} / \mathrm{cm}$, and a temperature of $25-$ $29^{\circ} \mathrm{C}$. We fed the fish live black worms four times per week. All procedures were in accordance with the guidelines established by the National Institutes of Health and were approved by the Institutional Animal Care and Use Committee at Washington University in St. Louis.

Surgery. The surgical procedure has been described previously (Carlson, 2009; Lyons-Warren et al., 2013b). Briefly, fish were anesthetized in a solution of $300 \mathrm{mg} / \mathrm{L}$ tricaine methanesulfonate (MS-222) and paralyzed with an intramuscular injection of $100 \mu \mathrm{l}$ of $3 \mathrm{mg} / \mathrm{ml}$ gallamine triethiodide (Flaxedil). We then respired the fish with $100 \mathrm{mg} / \mathrm{L}$ MS-222. Before surgery, we applied a drop of $0.4 \%$ lidocaine local anesthetic to the incision site. Next, we glued a head post to the skull and performed a craniotomy to expose ELp. Following surgery, we switched respiration to freshwater and allowed the fish to recover from anesthesia before beginning the recording session. We monitored the anesthetized state of the fish with a pair of electrodes external to the electric organ to record the fictive EOD generated by the electromotor neurons (Carlson, 2009). The fictive EOD is silenced during anesthesia; the return of fictive discharges at regular intervals signals that the fish has recovered. At the end of the recording session, the fish was anesthetized with $100 \mathrm{mg} / \mathrm{L} \mathrm{MS}-222$ respiration until no fictive EOD could be recorded, and then the fish was killed by freezing.

Intracellular whole-cell recordings. We obtained intracellular wholecell current-clamp recordings following previously published methods (Rose and Fortune, 1996; Carlson, 2009). We filled glass micropipettes of $20-40 \mathrm{M} \Omega$ resistance with a tip solution containing the following (in $\mathrm{mm}$ ): $100 \mathrm{CH}_{3} \mathrm{CO}_{2} \mathrm{~K}, 2 \mathrm{KCl}, 1 \mathrm{MgCl}_{2}, 5$ EGTA, 10 HEPES, $20 \mathrm{KOH}$, and 43 biocytin. We filled the pipette shank with the same solution except that we replaced biocytin with D-mannitol (Carlson, 2009). Initial seals were $>1 \mathrm{G} \Omega$. Intracellular recordings were amplified $10 \times$ and low-pass filtered (cutoff frequency, $10 \mathrm{kHz}$ ) through an Axopatch 200B amplifier (Molecular Devices) and digitized at a sampling rate of $97.7 \mathrm{kHz}$ (model RX8, Tucker-Davis Technologies). We saved recordings using custommade software for Matlab 7 (MathWorks).

Stimulus presentation. We delivered electrosensory stimuli through electrodes positioned at the perimeter of the recording chamber. For transverse stimulation to the fish, stimuli were delivered between three vertically oriented electrodes on one side of the chamber and three vertically oriented electrodes on the other side of the chamber. For longitudinal stimulation to the fish, stimuli were delivered between two vertically oriented electrodes on the front of the chamber and two vertically oriented electrodes on the back of the chamber (Lyons-Warren et al., 2013b). Stimuli consisted of monophasic square electric pulses. After establishing a recording, we first stimulated with single pulses while varying the pulse duration $(0.5-1.5 \mathrm{~ms})$, intensity $(1-100 \mathrm{mV} / \mathrm{cm})$, polarity (normal or reversed), and orientation (transverse or longitudinal to the fish) to identify the combination of features that elicited maximal post- synaptic potential (PSP) responses from a given neuron. We then delivered all stimuli to that neuron using this combination of features, varying only the interpulse intervals (Carlson, 2009). We discarded responses to stimulus pulses delivered $2-5 \mathrm{~ms}$ after a fictive EOD, since a corollary discharge at the level of the hindbrain blocks sensory responses occurring within this window (Bell and Grant, 1989).

Data analysis. We removed spikes from recording traces using a linear extrapolation method (Hedwig and Knepper, 1992). First, we smoothed the derivative of the recorded trace using a moving average filter with width of $0.5 \mathrm{~ms}$. We defined spike start as the point where the smoothed derivative first exceeded the prestimulus mean +4 SDs, and spike end as the point where the smoothed derivative decreased below the prestimulus mean -1 SD. We chose 4 SDs for the spike start to avoid identifying fast PSPs as spikes, and 1 SD for the spike end to ensure capturing as much of the spike as possible. If the smoothed derivative did not cross the spike end threshold, we used the first minimum in the smoothed derivative up to $8 \mathrm{~ms}$ after the spike start as the spike end. Such a wide window was necessary to accommodate variation in neuron responses, which depend not only on intrinsic membrane properties but also on the location on the membrane where the patch recording is located. To remove the spikes, we linearly extrapolated the membrane potential between spike start and spike end.

We averaged responses to 10 repetitions of each stimulus presented. Next, we determined the resting potential (RP) of the membrane by averaging the potential over a $50 \mathrm{~ms}$ window immediately preceding the stimulus. We measured the PSP amplitude by finding the maximum potential in a window starting $3 \mathrm{~ms}$ after stimulus pulse offset and ending immediately before the onset of the next pulse. We chose $3 \mathrm{~ms}$ as the start of the measurement window because the latency of electrosensory responses in ELa, the primary input to ELp, is $\sim 3 \mathrm{~ms}$ (Amagai, 1998; Carlson, 2009). If the maximum potential occurred after delivery of the next pulse, we increased the measurement window by $3 \mathrm{~ms}$ while ignoring the stimulus artifact. We subtracted the RP from the maximum potential to yield the PSP amplitude. For presentation purposes, we removed the stimulus artifact by linearly extrapolating the membrane potential from the time of pulse onset to $0.5 \mathrm{~ms}$ after pulse offset.

We assessed tuning to IPIs in 72 neurons using scanning IPI stimuli. We presented two scan stimuli; one consisted of a sequence of decreasing then increasing intervals (200-10 ms IPIs followed by $10-200 \mathrm{~ms}$ IPIs; see Fig. 1), and the other consisted of a sequence of increasing then decreasing intervals. These IPI scan stimuli are similar to frequency sweeps used to quantify response properties of the central auditory systems of many animals (e.g., Carrasco and Lomber, 2011; Williams and Fuzessery, 2012; Geis and Borst, 2013). We measured the PSPs elicited by each pulse in the two scan stimuli, and then averaged the PSPs in response to the same IPI. We also evaluated IPI tuning in nine neurons using trains of 10 pulses delivered at a constant IPI ranging from 10 to 100 $\mathrm{ms}$. We averaged responses to the 2 nd to 10 th pulses in the train to obtain the response of the neuron to each IPI (Carlson, 2009; George et al., 2011; Ma et al., 2013). We collected both scanning IPI and constant IPI tuning curves from two neurons, and in both cases the scanning and constant IPI tuning curves were in agreement with one another.

High-pass neurons respond best to short intervals, low-pass neurons respond best to long intervals, bandpass neurons respond best to intermediate intervals, and bandstop neurons respond best to short and long, but not intermediate, intervals (Carlson, 2009). To generate tuning curves, we normalized responses to each IPI by dividing by the maximum response (Fig. 1). If PSPs to all IPIs were negative (i.e., below the RP of the neuron), we multiplied all PSPs by -1 before normalizing by the maximum response. If the normalized responses to all IPIs remained at $>0.85$, we classified the neuron as all-pass (Carlson, 2009; George et al., 2011; Ma et al., 2013). Otherwise, we fit tuning curves with both Gaussian and sigmoidal functions to characterize IPI tuning (Groh et al., 2003). If the $r^{2}$ values of both the Gaussian and the sigmoidal fits were $<0.5$, we classified the tuning of the neuron as complex. Since Gaussian and sigmoidal curves can fit low-pass and high-pass tuning curves equally well, we used an $r_{\text {sigmoid }}^{2} / r_{\text {Gaussian }}^{2}$ ratio of 0.85 as a cutoff for comparing the goodness of the sigmoid and Gaussian fits (Groh et al., 2003). If at least one $r^{2}$ value was $\geq 0.5$, we used the ratio of the $r^{2}$ values to determine 
tuning. If the $r_{\text {sigmoid }}^{2} / r_{\text {Gaussian }}^{2}$ ratio was $\geq 0.85$, we classified the neuron as high-pass if the ratio of the sigmoid slope to the sigmoid amplitude was negative, and low-pass if this ratio was positive. If the $r_{\text {sigmoid }}^{2} / r_{\text {Gaussian }}^{2}$ ratio was $<0.85$, we classified the neuron as bandpass if the Gaussian amplitude was positive and bandstop if the Gaussian amplitude was negative. For tuning curves in which all PSPs were originally negative, we classified tuning as the opposite of the result of the fits to account for the reversed sign.

From the scanning IPI stimuli used to assess tuning, we also quantified the degree of sensitivity to the direction of IPI change. Changes in IPI are common characteristics of mormyrid electric communication signals (Carlson, 2002; Carlson and Hopkins, 2004; Wong and Hopkins, 2007). To quantify the degree of sensitivity to the direction of IPI change, we calculated a scan direction selectivity index (DSI). We started by normalizing the PSPs elicited by a scan stimulus to the maximum PSP. If the maximum potential in response to a particular IPI was below the RP of the neuron, we set the response to that IPI to 0 . This step was necessary to limit the range of the DSI from 0 to 1 . For a single scan stimulus, we next found the difference between the PSP evoked by a given IPI when presented during the decreasing portion of the scan, and that evoked by the same IPI when presented during the increasing portion of the scan. We took the sum of the absolute values of these differences across all IPIs and divided by the total number of IPIs ( $n=12$ IPIs). We then averaged the DSI across the two scan stimuli (increasing then decreasing scan stimulus, and decreasing then increasing scan stimulus). When the PSPs in response to each IPI are identical, regardless of scan direction, the DSI equals 0 (no directional selectivity); and when the PSPs in response to each IPI are equal to the maximum response in one direction and zero in the other direction, the DSI equals 1 (maximal directional selectivity)

To investigate the influence of temporal summation on IPI tuning and scan directional selectivity, we removed its effects from our PSP measurements. From the maximum potential evoked by each stimulus pulse, we subtracted the minimum potential that occurred between the time of the maximum potential and the time of the stimulus pulse. The results provide an estimate of the membrane potential changes evoked only by each pulse while minimizing the lingering effects of responses to previous pulses. To assess the validity of this method, we compared the results to measurements of true summation for the second PSP in 10 and 100 ms IPI trains. To measure true summation, we subtracted the change in membrane potential of the single-pulse response at the time of the second PSP from the amplitude of the second PSP. We then normalized the second PSP amplitudes resulting from each summation removal method to the first PSP amplitude in the train. We compared the results of the two methods in 40 neurons for which responses to both 10 and 100 ms IPIs were available. Our summation removal method resulted in three outlier neurons in which the 10 ms IPI PSPs were greatly overesti-

A

stimulus |

B

C
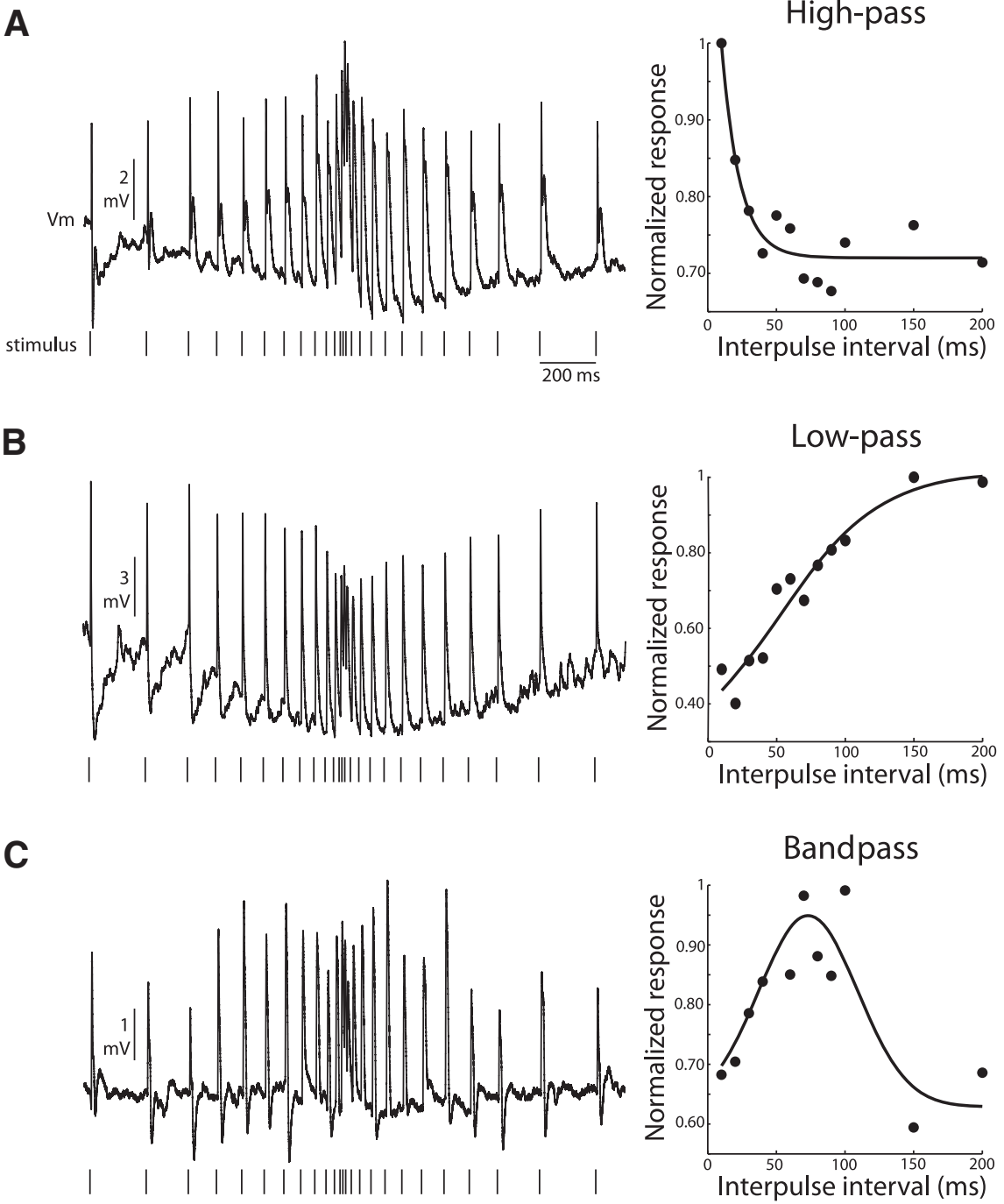

Bandpass
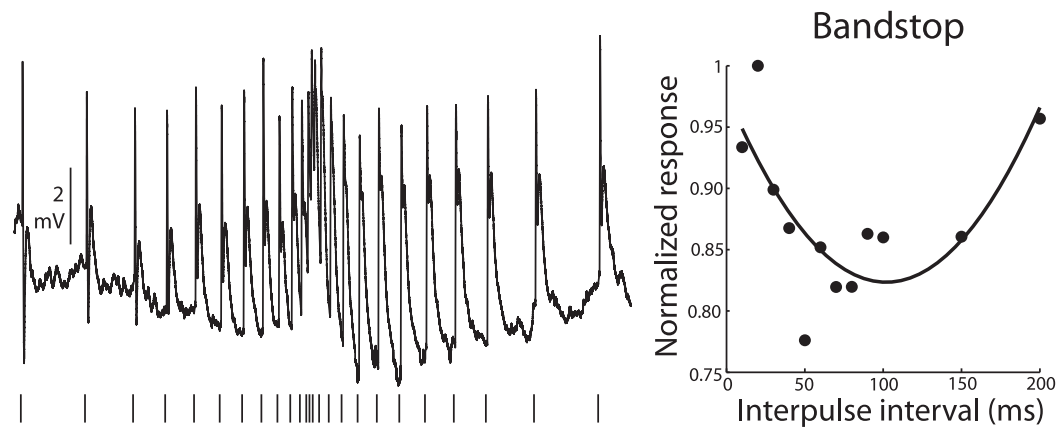

Figure 1. ELp neurons vary in their tuning to stimulus interval. $A-D$, Recorded $V_{m}$ values in response to a scan of decreasing then increasing IPIs (left), and tuning curve (right) for a high-pass $(\boldsymbol{A})$, low-pass $(\boldsymbol{B})$, bandpass $(\boldsymbol{C})$, and bandstop (D) neuron. Tick marks below the recording traces indicate stimulus times. Tuning curves were generated by averaging PSP amplitudes in response to the same IPI during a decreasing-increasing train and an increasing-decreasing train, then normalizing by the maximum average response. These curves were then fit with both a Gaussian and a sigmoidal function. Neurons were classified as high-pass or low-pass if the $r_{\text {sigmoid }}^{2} / r_{\text {Gaussian }}^{2}$ ratio was $\geq 0.85$, and as bandpass or bandstop if this ratio was $<0.85$ (see Materials and Methods). The sigmoidal (high-pass and low-pass) or Gaussian (bandpass and bandstop) fit is shown for each neuron.

mated, and one outlier neuron in which the $100 \mathrm{~ms}$ IPI PSPs were greatly overestimated relative to the true summation removal method. After removing these outliers, we found a linear correlation between the normalized PSPs using our summation removal method and the normalized PSPs using the true summation removal method at $10 \mathrm{~ms}$ IPIs $(y=0.54 x$ $\left.+0.88, r^{2}=0.24, F_{(1,34)}=11, p=0.0025\right)$ as well as $100 \mathrm{~ms}$ IPIs 
$\left(y=0.46 x+0.66, r^{2}=0.28, F_{(1,34)}=13, p=0.00099\right)$. Based on these results, we performed our summation removal method on the PSPs used to determine the IPI tuning and scan directional selectivity for each neuron to determine the contribution of temporal summation to these two properties. We did not include the four outlier neurons in this analysis.

In general, our summation removal method (median normalized PSP, 1.25; range, $0-4.2$ ) tended to modestly overestimate $10 \mathrm{~ms}$ IPI PSPs relative to the true summation removal method (median normalized PSP, 0.93 ; range, -0.09 to 4.0 ; Wilcoxon matched-pairs test, $Z_{(36)}=2.3$, $p=0.03)$. There was no difference between the results of the two methods for $100 \mathrm{~ms}$ IPIs (Wilcoxon matched-pairs test, $Z_{(36)}=1.7, p=$ $0.084)$. Therefore, our method of removing summation may overestimate PSP amplitudes at short intervals but not at long intervals. The net effect of this error would be a tendency to exaggerate the high-pass nature of summation-removed responses. Since we found that minimizing the effects of summation reduced the number of high-pass neurons (see Fig. 8 ), the possibility that we overestimated the high-pass characteristics of neurons means that there may have actually been fewer high-pass responses if we could have more accurately assessed tuning without summation. Thus, this error is conservative, as accounting for this modest overestimation could only lend added support to our conclusion that temporal summation is important for producing high-pass responses (see Results).

Synaptic conductance estimation. We estimated the synaptic conductances underlying neural responses following established methods (Wehr and Zador, 2003; Priebe and Ferster, 2005; Higley and Contreras, 2006; Monier et al., 2008; Gittelman et al., 2009), which are based on solving the following membrane equation:

$$
C_{m} \frac{d V_{m}}{d t}=g_{e}\left(V_{m}-V_{e}\right)+g_{i}\left(V_{m}-V_{i}\right)+g_{\text {leak }}\left(V_{m}-V_{\text {rest }}\right)+I_{\text {inj }},
$$

where $C_{m}$ is the membrane capacitance; $V_{m}$ is the membrane potential; $V_{\text {rest }}$ is the resting membrane potential; $I_{\text {inj }}$ is the injected current; $V_{e}$ and $V_{i}$ are the excitatory and inhibitory reversal potentials, respectively; and $g_{e}, g_{i}$, and $g_{\text {leak }}$ are the excitatory, inhibitory, and leak conductances, respectively.

We first measured the input resistance $\left(R_{m}\right)$ and membrane time constant $\left(\tau_{m}\right)$ by fitting a double exponential to the response of each neuron to a $100 \mathrm{~ms},-0.10 \mathrm{nA}$ current pulse, as described by Gittelman et al. (2009). We then used these values to calculate the membrane capacitance $\left(C_{m}=\tau_{m} / R_{m}\right)$.

Next, we obtained intracellular recordings of responses to single stimulus pulses, constant IPI trains (10 and $100 \mathrm{~ms}$ IPIs), and scanning IPI trains (10-200 ms IPIs) at multiple levels of current clamp (0 to -0.20 $\mathrm{nA})$. We estimated the capacitive current $\left(I_{\text {cap }}\right)$ according to the following equation:

$$
I_{\text {cap }}=C_{m} \frac{d V_{m}}{d t}
$$

Using the equation

$$
V_{m}=\frac{1}{g_{T}}\left(I_{\text {inj }}-I_{\text {cap }}\right)+V_{\text {rev }}
$$

where $g_{T}$ is the total conductance and $V_{\text {rev }}$ is the synaptic reversal potential, we created plots of $\left(I_{\text {inj }}-I_{\text {cap }}\right)$ versus $V_{m}$ for the different levels of current clamp at each time point in the recording. The inverse of the slope of the best-fit line at each time point yields $g_{T}$. The baseline $g_{T}$ represents $g_{\text {leak }}$, and was found by taking the inverse of the median slope of best fits over the $50 \mathrm{~ms}$ prestimulus period. We then calculated the synaptic conductance $\left(g_{\text {syn }}\right)$ throughout the recording as $g_{T}-g_{\text {leak }}$.

Next, we defined a baseline $\left(I_{\text {inj }}-I_{\text {cap }}\right)$ versus $V_{m}$ curve, with $y$-intercept equal to the median of the $y$-intercepts of the best fits during the prestimulus period, and slope equal to the inverse of $g_{\text {leak }}$. The intersection of the baseline curve with the best-fit line at each time point during the recording yields $V_{\text {rev }}$ at that time point. Following the simplification that the synaptic current is $0 \mathrm{nA}$ at $V_{\text {rev }}$, equations for $g_{e}$ and $g_{i}$ can be written as follows:

$$
g_{e}=g_{\text {syn }} \frac{\left(V_{i}-V_{\text {rev }}\right)}{\left(V_{i}-V_{e}\right)}
$$

and

$$
g_{i}=g_{\text {syn }} \frac{\left(V_{e}-V_{\mathrm{rev}}\right)}{\left(V_{e}-V_{i}\right)} .
$$

We used a $V_{e}$ of $0 \mathrm{mV}$ and a $V_{i}$ of $-106 \mathrm{mV}$, which was the calculated reversal potential for potassium based on our intracellular electrode solution and extracellular Hickman's Ringer's solution.

Because this method is based on the cell's $I-V$ linearity, spikes necessarily violate this linearity and cause error in conductance estimation that cannot be overcome through spike removal (Guillamon et al., 2006). Therefore, we discarded current-clamp levels containing spikes. However, if fewer than three current-clamp levels were spike free for a given neuron, we removed spikes by ignoring the portion of the recording trace from $2 \mathrm{~ms}$ before spike start to $2 \mathrm{~ms}$ after spike end before averaging across 10 stimulus repetitions. Spike start and end criteria were as described for spike removal. If this process resulted in gaps (due to the presence of spikes in all repetitions) or large edge effects (due to throwing out portions of some repetitions), we excluded the file from synaptic conductance estimation. If fewer than three current-clamp levels passed these criteria, we excluded the neuron from synaptic conductance estimation. An additional inclusion criterion required an RP of at least -40 $\mathrm{mV}$ at $0 \mathrm{nA}$ current injection.

Recordings from 48 of 83 neurons passed our inclusion criteria. The excluded recordings contained neurons from all tuning classes, and there were no differences in the tuning distributions of excluded versus included neurons $\left(\chi_{(5)}^{2}=6.3, p>0.25\right)$. Furthermore, there were no differences between included and excluded recordings in terms of resting membrane potential (Student's $t$ test, $t_{(81)}=1.9, p=0.068$ ), membrane time constant (Student's $t$ test, $t_{(81)}=-1.8, p=0.077$ ), or input resistance (Student's $t$ test, $t_{(81)}=-0.87, p=0.39$ ). We therefore conclude that our inclusion criteria did not bias the tuning or the passive membrane properties of the neurons analyzed in this study.

We median filtered synaptic conductances with a filter width of $1 \mathrm{~ms}$. To assess the goodness of the linear fits giving rise to the conductance estimates, we calculated the skewness of the linear $r^{2}$ distribution for each estimate according to the following equation:

$$
\text { skewness }(X)=\frac{n}{(n-1)(n-2)} \sum_{i=1}^{n}\left(\frac{X_{i}-\bar{X}}{\sigma}\right)^{3} \text {, }
$$

where $n$ is the number of points, $\bar{X}$ is the mean, and $\sigma$ is the SD of the $r^{2}$ distribution. Distributions clustered near 1 had negative skewness values, whereas distributions clustered near 0 had positive skewness values. If the skewness was $>-1$, we used only those conductance values at points where the $r^{2}$ of the $I-V$ fits were greater than the median $r^{2}$ value. Skewness values ranged from -40 to 0.9 across all conductance estimates, with $20 \%$ of estimates having skewness values -1 .

In several neurons ( $n=10$ neurons), the median-filtered synaptic reversal potential decreased below the inhibitory reversal potential, most likely due to a poor space clamp. In these cases, we set $g_{e}=0 \mathrm{nS}$ and $g_{i}=$ $g_{s y n}$ at the points where $V_{\text {rev }}$ was less than $V_{i}$, since the responses at these points were most likely dominated by inhibition.

Conductance estimations are based on a linear, isopotential neuron. While this method may cause slight underestimation of conductance magnitudes, it should not affect the time course (Wehr and Zador, 2003). We have tried to minimize these effects by comparing excitatory and inhibitory conductances within the same neuron, and by normalizing conductance magnitudes before comparisons across neurons. Further, although cable attenuation may cause inhibitory conductances to be slightly more underestimated than excitatory conductances (Wehr and Zador, 2003), we focused on differences between neuron types. Therefore, our comparisons should hold despite any systematic errors in estimation. Another potential source of error in our estimates would be the presence of voltage-gated conductances. Although we have no definitive evidence for voltage-gated conductances in ELp, we have tried to mini- 
mize potential effects by limiting our analysis to points in which the current-voltage relationship was approximately linear.

To quantify synaptic conductances, we measured the peak magnitudes evoked by stimulus pulses as the maximum conductance in a window starting $3 \mathrm{~ms}$ after stimulus pulse offset to immediately before onset of the next pulse. To determine onset and offset latencies of responses to single pulses, we first smoothed conductances using a median filter with a width of $2 \mathrm{~ms}$. Then the point at which the filtered conductance exceeded the prestimulus mean +3 SDs defined the onset, and the point at which the filtered conductance decreased below the prestimulus mean +1 SD defined the offset. In some cases, two phases of conductance increases were present. If the filtered conductance crossed the onset threshold $\leq 5 \mathrm{~ms}$ after the offset of the first phase, we recorded the onset and offset of a second phase. We measured conductance duration by subtracting conductance onset from offset; in neurons with two phases, we measured the duration as the offset of the second phase minus the onset of the first phase. For presentation purposes, we removed the stimulus artifact by linearly extrapolating the conductance estimates from the time of stimulus onset to $0.5 \mathrm{~ms}$ after stimulus offset.

To estimate the dynamics of short-term depression, we removed the effects of temporal summation from conductance estimates. From each conductance peak elicited by a stimulus pulse, we subtracted the minimum conductance that occurred between the time of the peak and the time of the preceding peak, starting with the second pulse in a train. The results provide an estimate of the conductance changes evoked only by each pulse while minimizing the lingering effects of responses to previous pulses. To assess the validity of this method, we compared the results to measurements of true summation for the second conductance peak in 10 ms IPI trains. To measure true summation, we subtracted the conductance value of the single-pulse response that occurred at the time of the second conductance peak elicited by the $10 \mathrm{~ms}$ IPI train from the second conductance peak. We then normalized the second conductance amplitudes resulting from each summation removal method to the amplitude of the first peak conductance in the train. We found a linear correlation between the results of the two methods for $10 \mathrm{~ms}$ IPI excitatory conductances $\left(y=0.79 x+0.13, r^{2}=0.68, F_{(1,40)}=85, p<0.000001\right)$ and inhibitory conductances $\left(y=0.72 x+0.16, r^{2}=0.54, F_{(1,40)}=46\right.$, $p<0.000001$ ).

To quantify the time course and degree of response decay (estimated conductances) or depression (estimated conductances with effects of summation removed) evoked by $10 \mathrm{~ms}$ IPI stimulus trains, we first normalized peak conductances to the conductance evoked by the first pulse. We then fit the normalized conductances with a single exponential function described by the following equation:

$$
y=a e^{\frac{-t}{\tau}}+b
$$

where $t$ is the time of stimulus pulses. For our normalized data, which by definition equal 1 at $t=0 \mathrm{~ms}$, we can simplify this equation to the following:

$$
y=a\left(e^{\frac{-t}{\tau}}-1\right)+1
$$

The coefficient $(a)$ of the best fit gives a measure of the magnitude of decay/depression, and $\tau$ gives a measure of the time course of decay/ depression. We restricted $\tau$ to between 0 and $90 \mathrm{~ms}$, corresponding to the range of stimulus times used. We restricted $a$ to between 0 and 1 . If a fit resulted in $a=0$, indicating no decay, we did not use the corresponding $\tau$ value. This situation occurred in two bandpass neurons; however, after the effects of summation were removed, $a>0$. Since we limited the data in the fit to the stimulus times used, the resulting $a$ and $\tau$ values reflect the decay/depression observed during the presented stimuli, and not necessarily steady-state values. Conductances that decay or depress faster will have a smaller $\tau$ value, and conductances that decay or depress to a greater degree will have a larger $a$ value.

To study the effects of depression, we estimated what synaptic conductance responses would be in the absence of plasticity by convolving conductances evoked by single-pulse stimuli with IPI trains. First, for single-pulse conductances in which points with poor linear $I-V$ fits were discarded, we linearly interpolated between the remaining points to recover a trace with the original uniform sampling rate. Next, we defined the single-pulse conductances as the time point at which the stimulus occurred until the end of the recording $(200 \mathrm{~ms})$. We then convolved the single-pulse conductances with a $10 \mathrm{~ms}$ IPI train. To quantify the time course and degree of the resulting summation of conductances, we fit a single exponential described as follows:

$$
y=a\left(1-e^{\frac{-t}{\tau}}\right)+b
$$

to the normalized peak responses to a $10 \mathrm{~ms}$ IPI train, where $t$ is the time of stimulus pulses. For our normalized data, which by definition equal 1 at $t=0 \mathrm{~ms}$, the offset term $b$ is equal to 1 . The $a$ and $\tau$ values of the best fit give a measure of the degree and time course, respectively, of temporal summation. We restricted $\tau$ to between 0 and $90 \mathrm{~ms}$, based on the stimulus times used, and we restricted $a$ to between 0 and the maximum normalized conductance minus 1 .

To estimate IPI tuning in the absence of plasticity, we convolved single-pulse conductances with the same IPI stimuli used to characterize tuning in each neuron. We then put these conductances into the membrane equation to estimate the membrane potential that would result. We measured PSPs from the estimated membrane potential and characterized IPI tuning as described for recorded potentials.

Statistics. We performed all statistical tests in Statistica version 6.1 (StatSoft). Parametric tests included Student's $t$ test, paired $t$ test, oneway ANOVA, and repeated-measures ANOVA. Nonparametric tests included Wilcoxon matched-pairs test and observed versus expected frequency $\chi^{2}$ test.

\section{Results}

We obtained intracellular whole-cell recordings from 83 ELp neurons during the presentation of electrosensory pulse trains with IPIs ranging from 10 to $200 \mathrm{~ms}$ (Fig. 1). Recordings from 48 neurons yielded conductance estimates that fit our inclusion criteria (see Materials and Methods). Their tuning curves were classified as high-pass ( $n=15$ neurons), low-pass ( $n=23$ neurons), bandpass ( $n=4$ neurons), bandstop ( $n=3$ neurons), complex ( $n=2$ neurons), or all-pass ( $n=1$ neuron). We did not consider complex or all-pass tuned neurons in any subsequent analysis. We have included bandpass and bandstop neurons in population data to give an accurate representation of the diversity of responses of ELp neurons. However, we focused our analysis primarily on responses from high- and low-pass neurons since the majority of ELp neurons fall into one of these two categories (Carlson, 2009; George et al., 2011; Kohashi and Carlson, 2014).

High- and low-pass neurons responded differently to shortinterval stimulation. At short IPIs, high-pass neurons exhibited increased synaptic response amplitudes, whereas low-pass neurons exhibited decreased response amplitudes (Fig. 1; Carlson, 2009). In some cases, the responses of high- and low-pass neurons to long-interval stimuli also varied depending on the direction of interval change, as we describe in detail later in this article. A variety of synaptic mechanisms have been shown to give rise to such temporal sensitivity (Baker et al., 2013), including temporal summation (George et al., 2011; Rose et al., 2011), short-term depression (Fortune and Rose, 2000; Klyachko and Stevens, 2006), and facilitation (Klyachko and Stevens, 2006). The relative timing of excitatory and inhibitory inputs has also been hypothesized to play a role (Grothe, 1994; Edwards et al., 2008). Here we address the extent to which these various synaptic mechanisms interact to generate temporal filtering of behaviorally relevant synaptic input patterns in an intact sensory midbrain circuit. 
A

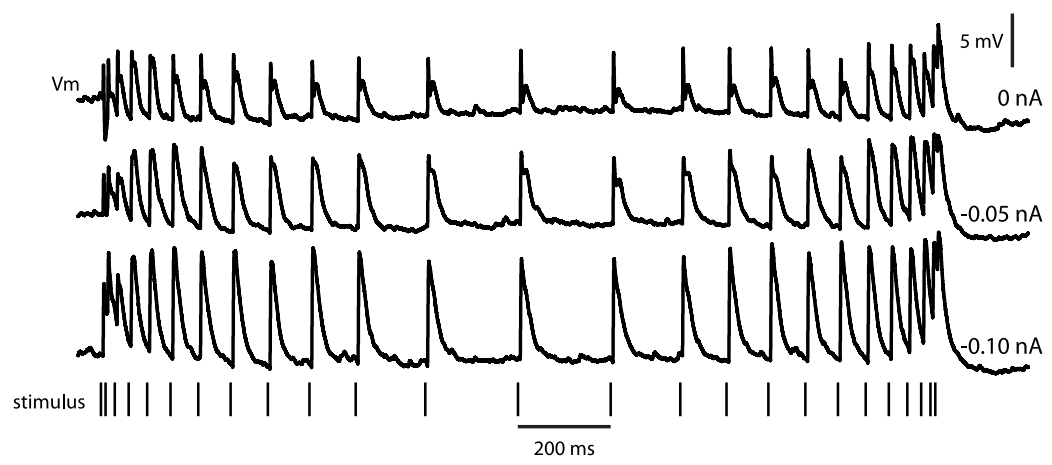

B
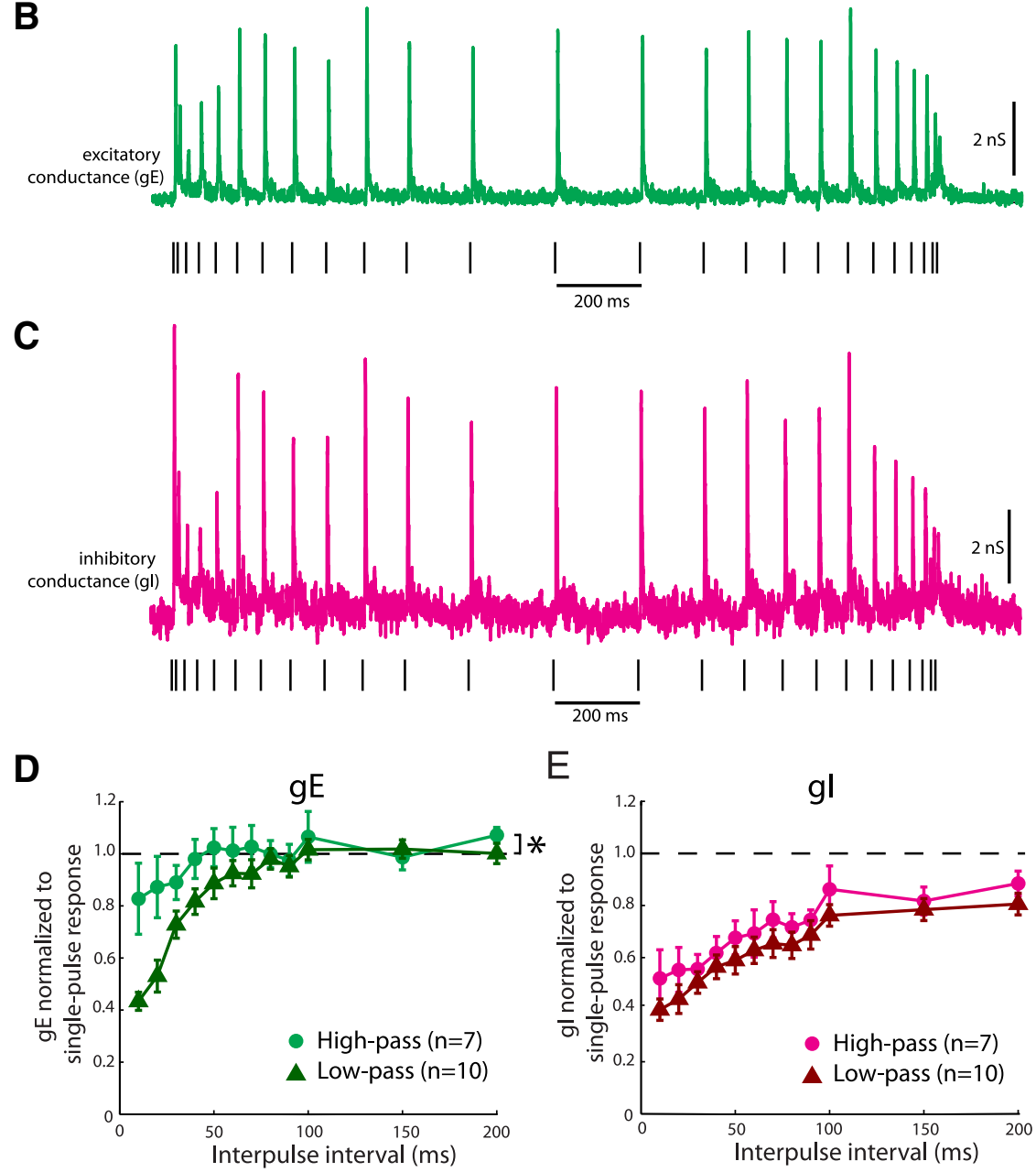

$\mathrm{E}$

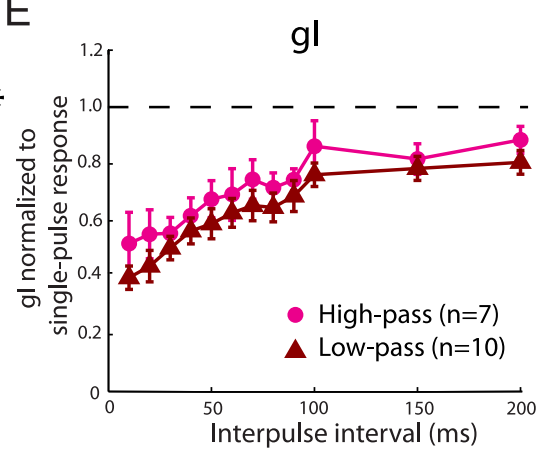

Figure 2. Excitatory and inhibitory conductances decrease at short intervals in response to IPI scans. $A$, The $V_{m}$ values recorded from a high-pass neuron during a decreasing-increasing IPI scan under three levels of current clamp. $\boldsymbol{B}, \boldsymbol{C}$, The excitatory $(\mathrm{gE} ; \boldsymbol{B})$ and inhibitory ( $\mathrm{gl} ; \boldsymbol{C})$ synaptic conductances estimated from the potentials recorded in $\boldsymbol{A}$. Notice that the peak amplitudes decrease at short IPIs. $\boldsymbol{D}, \boldsymbol{E}$, Average peak excitatory $(\boldsymbol{D})$ and inhibitory conductances $(\boldsymbol{E})$ normalized to single-pulse responses for high-pass and low-pass neurons. Error bars represent SEM. ${ }^{*} p<0.000001$, repeated-measures ANOVA, interaction between tuning and IPI. trosensory pulse trains of increasing and decreasing IPIs at multiple levels of holding current (Fig. $2 A$ ), and then estimated the underlying synaptic conductances (Fig. 2B,C). We found that the peak magnitude of both excitatory conductances (Fig. 2D) and inhibitory conductances (Fig. 2E) decreased at short intervals in all neurons, with the shortest intervals resulting in the smallest conductances.

Excitation was significantly more reduced at short IPIs in low-pass neurons compared with high-pass neurons (Fig. $2 D$; repeated-measures ANOVA, interaction between tuning and IPI, $F_{(11,15)}=6.3$, $p<0.000001)$. In contrast to excitation, inhibition was similarly reduced in the two groups of neurons during short IPIs (Fig. 2E; repeated-measures ANOVA, interaction between tuning and IPI, $F_{(11,15)}=$ $0.38 p=0.96)$. Notably, the amplitudes of inhibitory conductances were reduced compared with single-pulse responses, even at the longest IPI tested $(200 \mathrm{~ms})$ in both groups of neurons.

Since the differences between highand low-pass neurons were greatest at the shortest intervals tested, we next sought to characterize synaptic conductance dynamics during a train of 10 electrosensory pulses at a constant $10 \mathrm{~ms}$ IPI, which corresponds to approximately the minimum IPI that a single fish produces (Hopkins, 1986). We then compared these responses to the synaptic conductances evoked by 100 ms IPIs. In 42 ELp neurons, we recorded intracellular responses at multiple levels of holding current (Fig. 3A) and estimated the underlying synaptic conductances (Fig. 3B). A plot of normalized PSPs evoked by $10 \mathrm{~ms}$ IPIs reveals the diversity of responses of ELp neurons, with the PSPs of some neurons increasing or decreasing to various degrees (Fig. 3D). The synaptic conductance estimates resulting from these PSPs revealed widespread reduction of the magnitudes of both excitation and inhibition in neurons of all tuning classes (Fig. $3 E, F$ ). These decreases were rate-dependent, with greater conductance decreases during $10 \mathrm{~ms}$ IPIs compared with 100 ms IPIs (Fig. 3C, G-I).

\section{Excitatory and inhibitory synaptic conductances experience rate-dependent decreases in magnitude}

The increased PSP amplitudes at short intervals that characterize high-pass responses could result from weakened inhibition or strengthened excitation. Conversely, the decreased PSP amplitudes at short intervals that characterize low-pass responses could result from strengthened inhibition or weakened excitation. To reveal the excitatory and inhibitory conductances evoked by IPIs ranging from 10 to $200 \mathrm{~ms}$, we recorded intracellular responses of 7 high-pass and 10 low-pass neurons to elec-

\section{Inhibition depresses more than excitation in high- pass neurons}

We next investigated how the dynamics of decreasing excitation and inhibition related to IPI tuning. In general, high-pass neurons received inhibitory inputs that decreased more than excitatory inputs during the $10 \mathrm{~ms}$ IPI train (Fig. $4 B, C$; repeated-measures ANOVA, interaction between excitation/inhibition and pulse time, $\left.F_{(9,11)}=4.7, p=0.000031\right)$. To quantify the degree and time course of the decay of conductance magnitudes during $10 \mathrm{~ms}$ IPI trains, we fit the normalized peak conduc- 
A

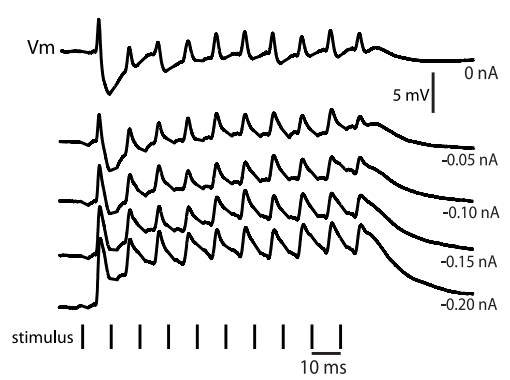

B
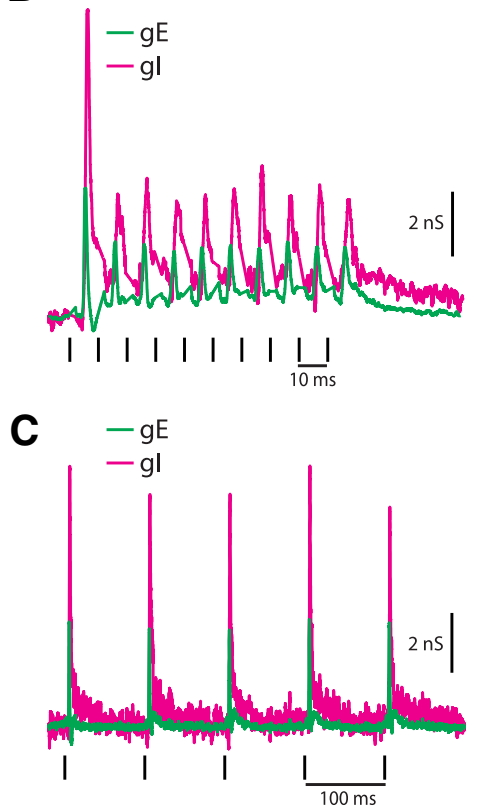

D

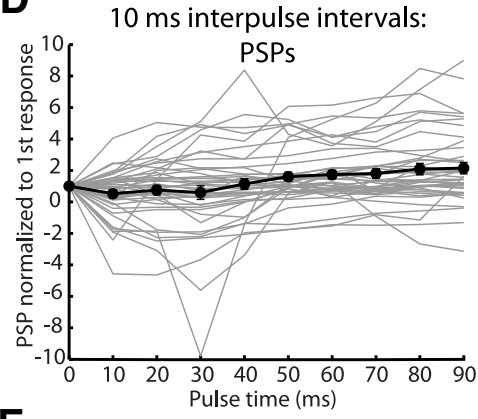

E

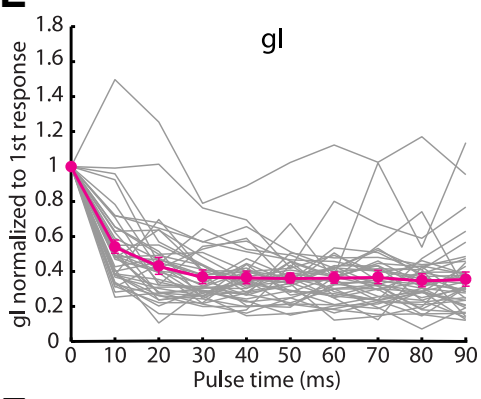

F

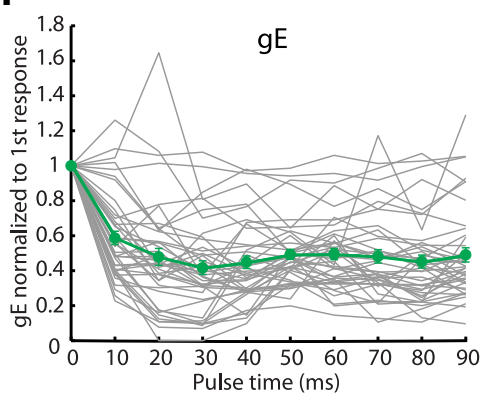

G
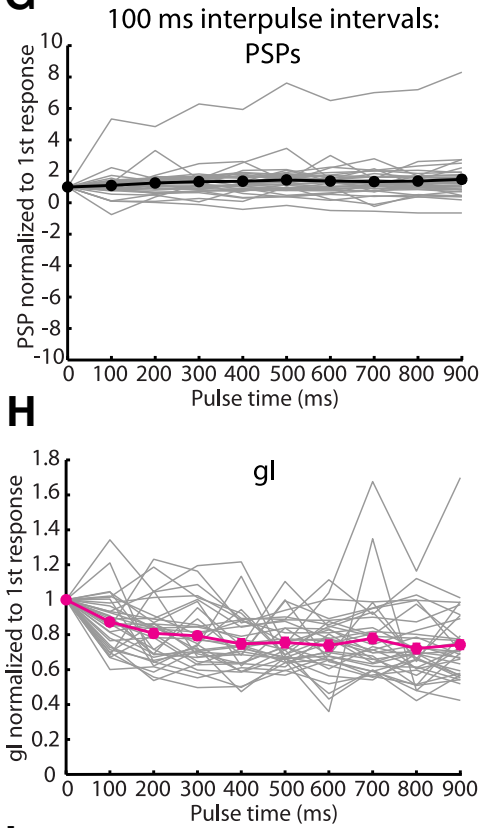

I

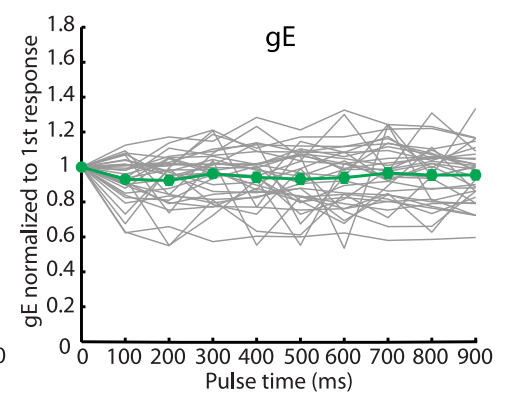

Figure 3. Interval-tuned neurons receive excitatory and inhibitory inputs that decrease to varying degrees during $10 \mathrm{~ms}$ IPIs. $\boldsymbol{A}$, The $V_{m}$ values recorded from a low-pass Elp neuron in response to a $10 \mathrm{~ms} \mathrm{IPI} \mathrm{train} \mathrm{under} \mathrm{multiple} \mathrm{levels} \mathrm{of} \mathrm{current} \mathrm{clamp.} \boldsymbol{B}$, Estimated excitatory $(\mathrm{gE})$ and inhibitory $(\mathrm{gl})$ conductances underlying the responses in $\boldsymbol{A}$. $\boldsymbol{C}$, Estimated synaptic conductances of the same neuron in $\boldsymbol{A}$ and $\boldsymbol{B}$ in response to a $100 \mathrm{~ms} I \mathrm{PI}$ train. $\boldsymbol{D}-\boldsymbol{F}$, Plots of normalized PSP $(\boldsymbol{D})$, inhibitory conductance $(\boldsymbol{E})$, and excitatory conductance $(\boldsymbol{F})$ amplitudes evoked by a $10 \mathrm{~ms} I \mathrm{PI}$ train versus stimulus time. Plots for each individual neuron ( $n=42$ neurons) are shown in gray, and the averages \pm SEM are shown in black (PSPs), magenta ( $\mathrm{gl}$ ), and green ( $\mathrm{gE}$ ). $\mathbf{G}-\mathbf{I}$, Same as in $\boldsymbol{D}-\boldsymbol{F}$ for the responses of 34 ELp neurons to $100 \mathrm{~ms}$ IPI trains.

tances evoked by the stimulus train with a single exponential function (see Materials and Methods). The resulting $\tau$ value gives a measure of how quickly the conductance decreased, with smaller $\tau$ values indicating faster decay. The amplitude $(a)$, or coefficient, of the fit gives a measure of the magnitude of decay, with larger $a$ values indicating decay to a greater degree.

The greater decrease in inhibition compared with excitation in high-pass neurons was due to inhibition that tended to decay faster (Fig. $4 E$, smaller $\tau$ values) and to a greater degree (Fig. $4 F$, larger $a$ values) than excitation. However, conductances rarely returned to baseline levels between the peaks evoked by each pulse in the $10 \mathrm{~ms}$ IPI train (Fig. 4B), indicating that temporal summation of conductance changes to previous pulses likely influenced the peak conductances. Therefore, to determine the extent to which summation affected the degree and time course of conductance changes, we removed the effects of summation from the 2 nd to 10 th peak conductances by subtracting the minimum conductance value occurring between the measured peak and the preceding peak. We then normalized these summation-removed peak values by the peak elicited by the first pulse in the train and fit these data with single exponential functions (see Materials and Methods). The results provide an estimate of the actual short- term depression of excitation and inhibition, with the confounding effects of temporal summation removed.

Inhibition depressed more than excitation in high-pass neurons (Fig. 4D; repeated-measures ANOVA, interaction between excitation/inhibition and pulse time, $\left.F_{(9,11)}=3.0, p=0.0036\right)$. Accordingly, inhibition tended to depress faster (Fig. 4E, smaller $\tau$ values) and to a greater degree (Fig. $4 F$, larger $a$ values) than excitation. Six of 13 high-pass neurons (46\%) received excitation that depressed more slowly and to a lesser degree than inhibition, 5 neurons (39\%) received excitation that depressed to a lesser degree than inhibition, and the remaining 2 neurons (15\%) received excitation that depressed more slowly than inhibition. Therefore, all high-pass neurons fit a depression model for high-pass tuning: with inhibition depressing more quickly and/or to a greater degree than excitation, depolarization of the neurons' membrane potentials increased with repeated stimulation (Fig. 4A).

These data also suggest a role for temporal summation of excitation in establishing high-pass responses. The differences between excitation and inhibition were greater for the normalized observed conductances (Fig. 4C) than for the normalized conductances after summation was removed (Fig. 4D). This find- 
A

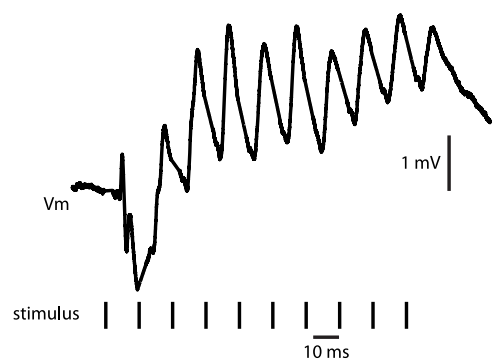

C

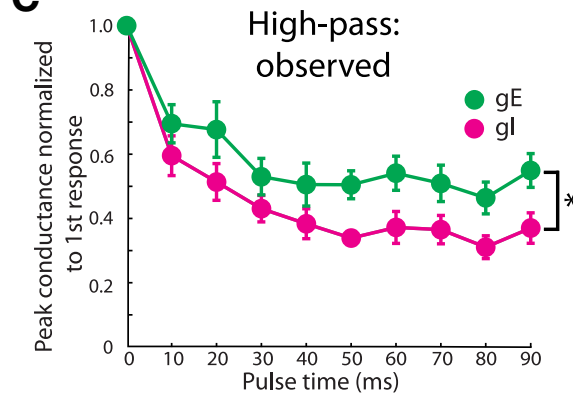

E

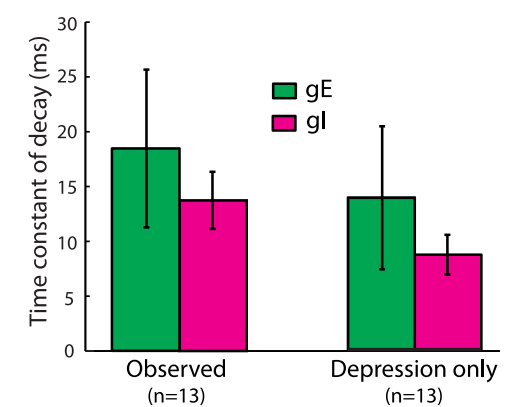

B

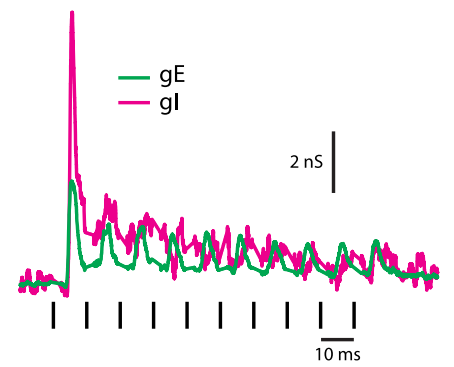

D

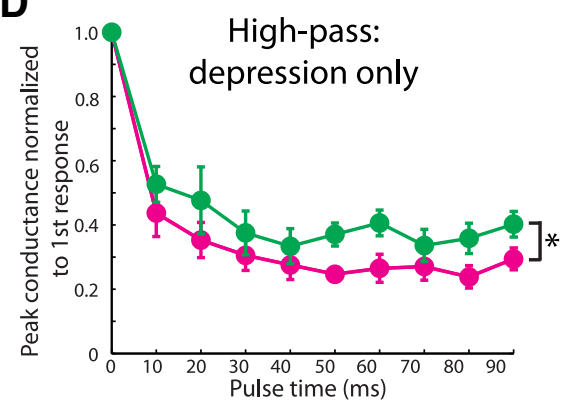

F

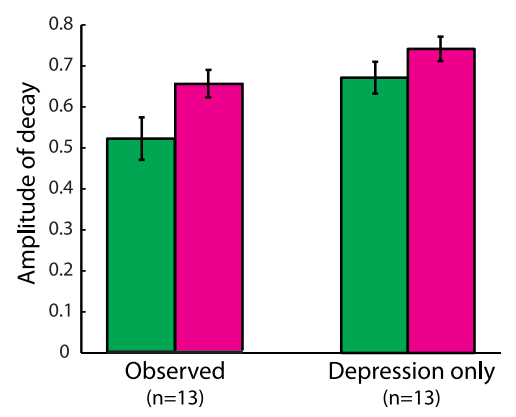

Figure 4. Excitation depresses less than inhibition at short intervals in high-pass neurons. $\boldsymbol{A}$, The $V_{m}$ values recorded from a representative high-pass neuron in response to a $10 \mathrm{~ms} I P I$ train. $\boldsymbol{B}$, The excitatory $(\mathrm{gE})$ and inhibitory $(\mathrm{gl})$ synaptic conductances underlying the responses shown in $\boldsymbol{A}$. C, Normalized synaptic conductances during a $10 \mathrm{~ms}$ IPI train in high-pass neurons ( $n=13$ neurons). ${ }^{*} p<0.0001$, repeated-measures ANOVA, interaction between excitation/inhibition and pulse time. $\boldsymbol{D}$, Normalized synaptic conductances during the $10 \mathrm{~ms}$ IPI train after removing the effects of temporal summation (see Materials and Methods) for high-pass neurons. ${ }^{*} p<0.01$, repeated-measures ANOVA, interaction between excitation/inhibition and pulse time. $\boldsymbol{E}, \boldsymbol{F}$, Bar graphs of average time constants $(\boldsymbol{E})$ and amplitudes $(\boldsymbol{F})$ of exponential fits to normalized observed and summation-removed (depression only) conductances during $10 \mathrm{~ms}$ IPI trains in high-pass neurons. Error bars represent SEM.

ing indicates that temporal summation must have contributed to the observed differences in excitation and inhibition shown in Figure $4 C$. Most likely, the slower depression of excitation (Fig. $4 E$ ) allowed for greater summation of excitation compared with inhibition during short IPIs, leading to excitation that decreased less overall than inhibition (Fig. 4C).

\section{Excitation depresses more than inhibition in most, but not all, low-pass neurons}

In general, low-pass neurons received excitatory and inhibitory inputs that decreased similarly during $10 \mathrm{~ms}$ IPIs (Fig. $5 B, C$; repeated-measures ANOVA, interaction between excitation/inhibition and pulse time, $\left.F_{(9,20)}=1.5, p=0.15\right)$. Excitation tended to decrease more quickly (Fig. $5 E$, smaller $\tau$ values) than inhibition, although excitation and inhibition decreased with similar amplitudes of decay (Fig. $5 F$ ) in low-pass neurons. However, we found that low-pass neurons varied in the relative balance of excitatory and inhibitory decreases. Seven of 22 low-pass neurons (32\%) received excitation that decreased more quickly $\left(\tau_{e}<\tau_{i}\right)$ and to a greater degree than inhibition $\left(a_{e}>a_{i}\right)$. Six neurons $(27 \%)$ received excitation that decreased more quickly than inhibition, and 3 neurons (14\%) received excitation that de- creased to a greater degree than inhibition. The remaining 6 low-pass neurons (27\%), however, received excitation that decreased more slowly $\left(\tau_{e}>\tau_{i}\right)$ and to a lesser degree than inhibition $\left(a_{e}<a_{i}\right)$.

After removing the effects of temporal summation, we found that excitation and inhibition tended to depress similarly in low-pass neurons during $10 \mathrm{~ms}$ IPIs (Fig. $5 D$; repeated-measures ANOVA, interaction between excitation/inhibition and pulse time, $\left.F_{(9,20)}=1.3, p=0.23\right)$. Accordingly, the depression time constants (Fig. 5E) and amplitudes (Fig. 5F) were similar for excitation and inhibition in low-pass neurons. However, low-pass neurons actually varied in the balance of relative depression of excitation and inhibition. Three of 22 low-pass neurons (14\%) received excitation that depressed more quickly and to a greater degree than inhibition, 6 neurons (27\%) received excitation that depressed more quickly than inhibition, and 5 neurons (23\%) received excitation that depressed to a greater degree than inhibition. Therefore, almost two-thirds of low-pass neurons fit a depression model for low-pass tuning: since excitation depressed to a greater degree and/or more quickly than inhibition, the membrane potentials of the neurons decreased with repeated stimulation (Fig. $5 A)$. The remaining 8 neurons (36\%), however, received excitation and inhibition that were inconsistent with this depression model for low-pass tuning. In these neurons, excitation depressed more slowly and to a lesser degree than inhibition. Factors other than differences in depression of excitatory and inhibitory pathways must therefore contribute to low-pass tuning in this group of neurons.

\section{Temporal summation of excitation contributes to high- pass tuning}

A previous in vitro and computational study of ELp neurons demonstrated a role for temporal summation of excitation and inhibition in establishing IPI tuning (George et al., 2011). Indeed, removing the effects of summation from observed conductance values revealed that summation of excitation contributed to differences in excitatory and inhibitory decreases during short-interval responses in high-pass neurons (Fig. 4, compare $D, C$ ). Temporal summation of excitatory or inhibitory conductances will occur if the durations of the respective conductance changes are longer than the stimulation interval. Therefore, excitation that lasts longer than inhibition would result in greater temporal summation of excitation at short intervals, which could contribute to high-pass responses. Likewise, inhibition that lasts longer than excitation would result in greater summation of inhibition, which could contribute to lowpass responses at short intervals (Baker et al., 2013).

The membrane capacitance of a neuron will cause PSPs to last longer than the underlying conductance changes, which will lead to further temporal summation. To investigate the role of the 
initial durations of excitation and inhibition in establishing interval tuning, we collected responses to single electrosensory stimulus pulses. Comparing the PSPs evoked by single pulses to those evoked by $10 \mathrm{~ms}$ IPI trains in high-pass neurons supports a role for temporal summation of excitation in producing larger-amplitude synaptic responses at short intervals (Fig. 6A). A single stimulus pulse elicits two depolarizing phases separated by a hyperpolarization. This kind of response was frequently observed in high-pass neurons. The initial depolarization evoked by the second pulse in the $10 \mathrm{~ms}$ train overlaps in time with the later depolarization evoked by the first pulse, resulting in a greater depolarization. In this way, temporal summation of depolarizing PSPs contributes to establishing high-pass responses.

To study the contributions of excitation and inhibition to these responses, we estimated the synaptic conductances underlying single-pulse responses in 13 high-pass neurons. We found that highpass neurons received excitatory and inhibitory inputs of similar durations (Fig. $6 D$; paired $t$ test, $t_{(12)}=-0.11, p=0.91$ ). These balanced durations indicate that the two conductances should summate at a similar range of IPIs, such that summation of excitation and inhibition partially counteract each other.

Interestingly, the amplitude of inhibition in response to single pulses was significantly larger than excitation in high-pass neurons (Fig. 6E; paired $t$ test, $\left.t_{(12)}=-2.8, p=0.016\right)$. Stronger inhibition than excitation in response to the onset of a stimulus train would serve to initially limit excitatory postsynaptic responses. However, in response to shortinterval stimulus trains, this strong inhibition will depress more than excitation (Fig. 4D), leading to high-pass tuning due to increased relative excitation that is further enhanced by the effects of temporal summation (Fig. 6A).

\section{Long-lasting onset inhibition contributes to low-pass tuning} Comparing the PSPs in response to $10 \mathrm{~ms}$ IPI trains to those elicited by single pulses in low-pass neurons reveals a potential role for long inhibition in establishing decreased synaptic responses at short intervals (Fig. 6B,C). Single pulses evoked an initial long-lasting hyperpolarization that drastically reduced the baseline potential on top of which subsequent PSPs occurred, resulting in a net hyperpolarization. The time course of recovery of PSPs during the stimulus train approximately followed the time course of the single-pulse hyperpolarization (Fig. $6 B, C$ ). These effects were seen in low-pass neurons that fit a low-pass depression model (i.e., excitation depressed faster and/or to a greater degree than inhibition in response to $10 \mathrm{~ms}$ IPIs; Fig. 6B). These same effects were also observed in low-pass neurons whose $10 \mathrm{~ms}$ IPI conductances did not fit a depression model (Fig. 6C). Synaptic conductance estimates of single-pulse responses in 22 low- pass neurons revealed that inhibition lasted significantly longer than excitation in low-pass neurons (Fig. $6 D$; paired $t$ test, $t_{(22)}=$ $-3.9, p=0.00083)$. There was no difference in the amplitudes of excitatory and inhibitory single-pulse conductances in low-pass neurons (Fig. $6 E$; paired $t$ test, $t_{(22)}=-0.05, p=0.96$ ).

On average, inhibition lasted $\sim 10 \mathrm{~ms}$ longer than excitation in low-pass neurons (Fig. 6D), indicating that inhibitory conductances should summate at slightly longer IPIs than excitatory conductances. This longer inhibition did not translate into greater effects of summation on inhibitory versus excitatory conductances at $10 \mathrm{~ms}$ IPIs, however (Fig. 5). Further, notice that after the long-lasting hyperpolarization in response to the first pulse in a $10 \mathrm{~ms}$ IPI train, PSPs of low-pass neurons gradually recovered instead of continuing to hyperpolarize as would occur due to summation if this long-lasting inhibition followed each pulse (Fig. $6 B, C$ ). It is likely that the strong depression of both excitation and inhibition in low-pass neurons (Fig. 5D, F) acts to limit the extent of temporal summation. Therefore, it appears that long-lasting onset inhibition that reduces excitatory responses to subsequent pulses is an important contributor to 
A

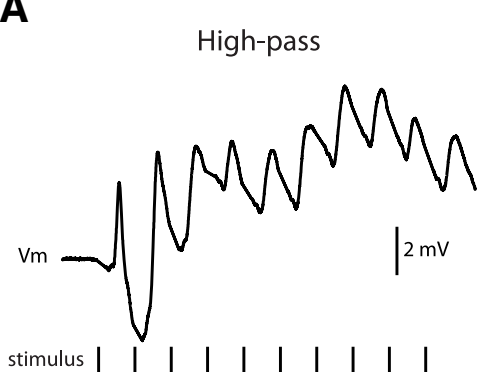

stimulus | | | | | | | | | $\frac{\mid \mathrm{l}}{10 \mathrm{~ms}}$

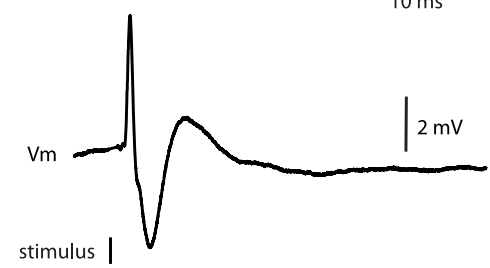

stimulus

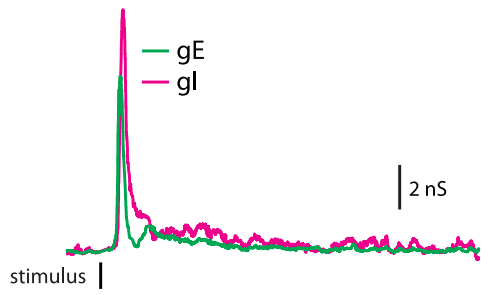

B

Conductances fit
low-pass depression model
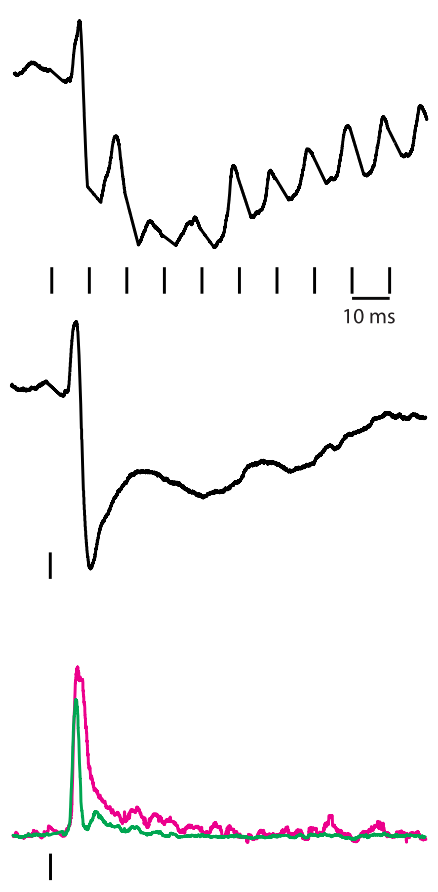

C

Conductances do not fit low-pass depression model
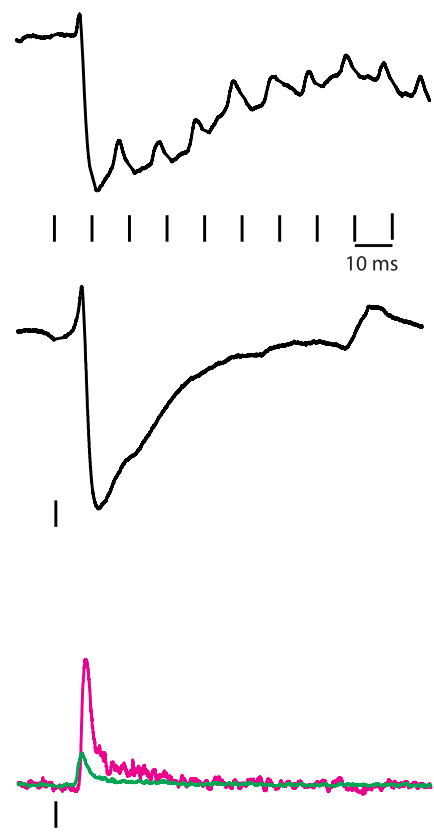

E

Single pulses
D

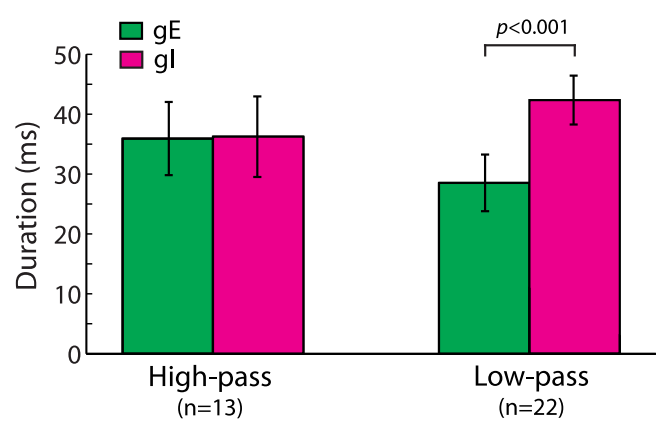

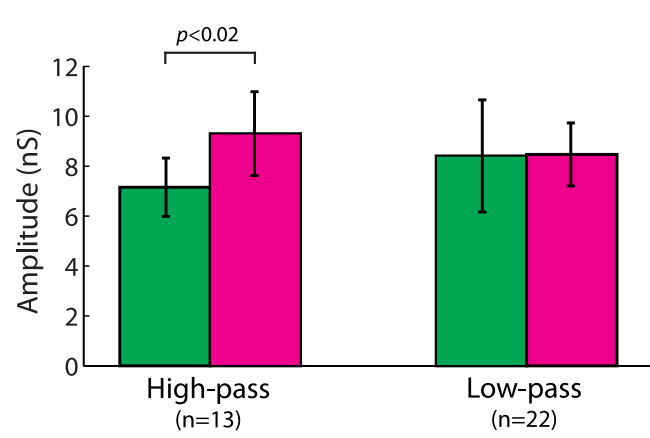

Figure 6. Temporal summation of excitation contributes to high-pass responses, whereas long onset inhibition contributes to low-pass responses. $A, V_{m}$ recordings in response to a $10 \mathrm{~ms} I P I$ train (top) and a single stimulus pulse (middle), and excitatory ( $\mathrm{gE}$ ) and inhibitory ( $\mathrm{gl}$ ) synaptic conductances (bottom) underlying the single-pulse PSPs for a representative high-pass neuron. $\boldsymbol{B}$, Same as in $\boldsymbol{A}$ for a low-pass neuron whose $10 \mathrm{~ms} \mathrm{IPI}$ conductances fit a depression model for low-pass tuning. $\boldsymbol{C}$, Same as in $\boldsymbol{A}$ for a low-pass neuron whose $10 \mathrm{~ms}$ IPI conductances do not fit a depression model for low-pass tuning. $\boldsymbol{D}, \boldsymbol{E}$, Bar graphs of the average durations $(\boldsymbol{D})$ and amplitudes $(\boldsymbol{E})$ of excitatory and inhibitory conductances elicited by single pulses in high- and low-pass neurons. Error bars represent SEM. The $p$ values are shown for significant differences between excitation and inhibition resulting from paired $t$ tests.

low-pass tuning, regardless of whether differences in short-term depression of excitation and inhibition enhance this tuning.

\section{Passive membrane properties do not play a major role in establishing interval tuning}

Differences in the effects of temporal summation between highand low-pass neurons could be influenced by passive membrane properties (Fortune and Rose, 1997b). We estimated the membrane time constant, capacitance, and input resistance of each neuron by fitting a double exponential to responses to a $100 \mathrm{~ms}$, $-0.10 \mathrm{nA}$ current step (see Materials and Methods). We found no differences in the membrane time constant (Student's $t$ test, $t_{(33)}=-0.49, p=0.63$; mean $\left.=7.5 \pm 0.6 \mathrm{~ms}\right)$, membrane capacitance (Student's $t$ test, $t_{(33)}=-0.087, p=0.93$; mean $=$ $74 \pm 6 \mathrm{pF}$ ), or input resistance (Student's $t$ test, $t_{(33)}=0.48$, $p=0.63$; mean $=119 \pm 11 \mathrm{M} \Omega$ ) between high- and low-pass neurons. Therefore, differences in the observed effects of sum- mation between high- and low-pass neurons are influenced primarily by the properties of their inputs and not by the passive properties of their membranes.

\section{Differences in the relative timing of excitation and inhibition do not contribute to interval tuning}

The relative timing of excitation and inhibition has been proposed to play a role in interval tuning (Grothe, 1994; Edwards et al., 2008; Baker et al., 2013). We compared the times of the excitatory and inhibitory peak conductances in response to each pulse in a $10 \mathrm{~ms}$ IPI train. Inhibitory conductances reached their maximum significantly later (by $\sim 0.6 \mathrm{~ms}$ ) than excitatory conductances (repeated-measures ANOVA, effect of excitation/inhibition, $\left.F_{(1)}=7.0, p=0.012\right)$ with no systematic differences between tuning groups (effect of tuning, $F_{(1)}=0.001, p=0.98$; interaction between tuning and excitation/inhibition, $F_{(1,31)}=$ $0.31, p=0.58$; interaction between tuning and pulse time, $F_{(9,15)}=$ 
0.40, $p=0.93)$. The latency of PSPs evoked by $10 \mathrm{~ms}$ IPI trains tended to increase with repeated stimulation (repeated-measures ANOVA, effect of pulse time, $F_{(9)}=2.0, p=0.039$ ), but there were no differences between highand low-pass neurons (effect of tuning, $F_{(1)}=0.89, p=0.35$; interaction between pulse time and tuning, $F_{(9,33)}=0.29, p=$ $0.98)$. While the relative timing of inputs certainly contributes to the responses of an individual neuron, we did not find evidence that systematic variation in relative timing plays a major role in establishing different patterns of interval tuning in ELp.

\section{Short-term depression is essential for diversity in interval tuning}

By subtracting the effects of temporal summation from excitatory and inhibitory conductances, we have demonstrated that summation of excitation contributes to producing high-pass responses (Fig. 4). To provide an estimate of what responses would occur in the absence of depression, we convolved single-pulse conductances with $10 \mathrm{~ms}$ IPI trains, and then used the membrane equation to estimate the resulting membrane potentials (see Materials and Methods). The results allowed us to investigate how temporal summation alone (without depression) would influence the responses of ELp neurons (Fig. 7). The membrane potentials resulting from convolved conductances typically decreased drastically with repeated stimulation (Fig. $7 A$ ). Convolutions of synaptic conductances resulted in excitation and inhibition that gradually increased due to temporal summation (Fig. $7 B$ ), with the extent of summation varying across neurons.

Without depression, excitation would summate significantly more than inhibition at $10 \mathrm{~ms}$ IPIs in high-pass neurons (Fig. 7C; repeatedmeasures ANOVA, interaction between excitation/inhibition and pulse time, $\left.F_{(9,11)}=2.7, p=0.0078\right)$. In contrast, inhibition would summate significantly more than excitation in low-pass neurons (Fig. 7D; repeated-measures ANOVA, interaction between excitation/inhibition and pulse time, $F_{(9,11)}=35, p<$ $0.001)$. However, the magnitude of the difference in summation of excitation versus inhibition was far greater for low-pass neurons than for high-pass neurons (Fig. 7, compare $D, C$ ), reflecting the longer onset inhibition of low-pass neurons (Fig. 6D).

To quantify the time course and degree of summation of the convolved conductances, we fit an exponential function to the normalized responses (see Materials and Methods). The resulting time constant of the fit is smaller for convolved conductances that summate faster, and the resulting amplitude of the fit is greater for convolved conductances that summate to a greater extent.

Convolved excitatory conductances tended to summate more quickly (Fig. 7E, smaller $\tau$ values) than inhibitory conductances in high-pass neurons, although the two conductances reached

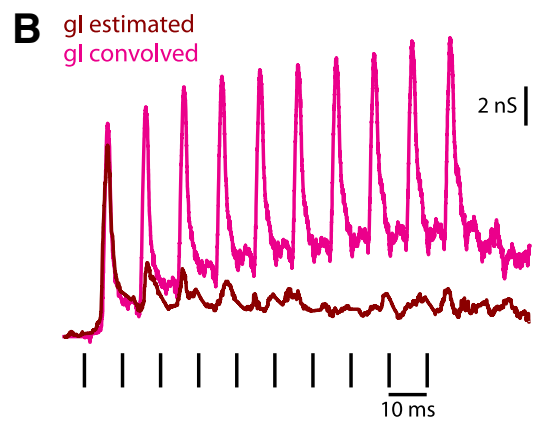

D Low-pass:

High-pass: ummation only

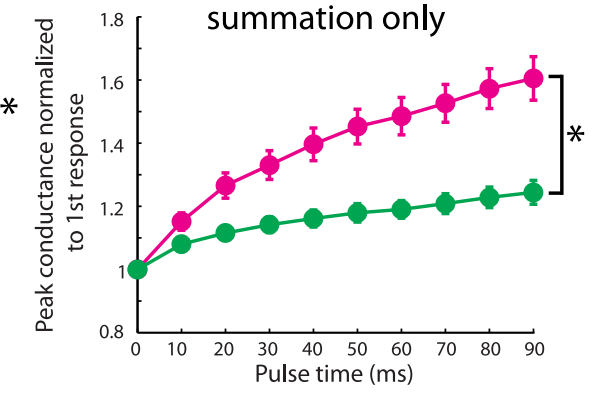

$\mathbf{F}$

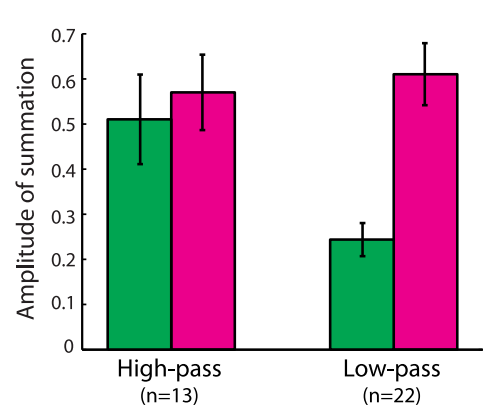

Figure 7. Convolutions of single-pulse conductances reveal that, without depression, excitation would summate more than inhibition in high-pass neurons, whereas inhibition would summate more than excitation in low-pass neurons. $A$, The recorded rane potential (" $V_{m}$ recorded") and the membrane potential resulting from conductance convolutions (" $V_{m}$ from convolu列 volved") inhibitory conductances of the same neuron in $A$ in response to a $10 \mathrm{~ms} \mathrm{IPI} \mathrm{train.} \boldsymbol{C}, \boldsymbol{D}$, Normalized convolved synaptic interaction between excitation/inhibition and pulse time. $\boldsymbol{E}, \boldsymbol{F}$, Bar graphs of the average time constants $(\boldsymbol{E})$ and amplitudes $(\boldsymbol{F})$ of single exponential fits to normalized convolved excitatory and inhibitory conductances. Error bars represent SEM.

a similar level of summation (Fig. $7 F$ ). In contrast, inhibition tended to summate to a greater extent (Fig. $7 F$, larger $a$ values) than excitation in low-pass neurons, even though the two inputs summated with similar time courses (Fig. $7 E$ ). These results are in line with the findings of the single-pulse balance of excitation and inhibition in the two groups of neurons. Low-pass neurons experienced longer inhibition that, in the absence of depression, would summate more than excitation. High-pass neurons received excitatory and inhibitory inputs of similar durations that, in the absence of depression, would summate approximately equally. However, the summation of onset inhibition that is of larger magnitude than onset excitation (Fig. 6E) would lead to inhibition-dominated responses in high-pass neurons.

Depression and temporal summation both contribute to diversity of IPI tuning

We have shown that both temporal summation and depression of excitation and inhibition contribute to the increasing synaptic response amplitudes of high-pass neurons and the decreasing 
synaptic response amplitudes of low-pass neurons at short intervals. Next, we sought to evaluate the relative contributions of each mechanism in generating the diversity of IPI tuning observed among ELp neurons. To reveal the contribution of depression to IPI tuning, we convolved single-pulse conductances with the same IPI stimuli used to characterize tuning in each neuron and then used the membrane equation to estimate the resulting membrane potentials. We then measured the PSPs of the estimated membrane potentials in response to each stimulus pulse, and generated tuning curves as described in Materials and Methods. The results provide an estimate of the tuning of each neuron in the absence of depression.

The averaged tuning curves of the responses without depression in both tuning groups decreased sharply at short intervals, with PSPs at the shortest intervals dropping below the resting potentials of neurons (Fig. $8 A, B$, "summation only" curves). The results therefore reveal the importance of short-term depression in establishing high-pass tuning. After removing the effects of depression, no neurons were classified as being high-pass. The majority of high-pass neurons ( $n=7$ of 12 neurons; 58\%) were classified as low-pass tuned, three neurons (25\%) were classified as bandpass tuned, and the remaining two neurons (17\%) were classified as bandstop tuned after removing depression. In contrast, the majority of low-pass neurons ( $n=14$ of 19 neurons; $74 \%)$ were still classified as low-pass tuned after removing depression. Of the remaining low-pass neurons, 3 (16\%) were classified as bandstop tuned, 1 (5\%) was classified as bandpass tuned, and $1(5 \%)$ was classified as complex tuned after removing depression. These results suggest that without depression, the balance of excitation and inhibition would produce primarily lowpass responses. Thus, removing the effects of depression caused a significant shift toward low-pass tuning and away from high-pass tuning among the population of ELp neurons (Fig. $8 C ; \chi_{(4)}^{2}=18$, $p<0.0013)$.

To evaluate the contribution of temporal summation to producing the observed diversity of IPI tuning, we minimized its effects by removing the lingering effects of responses to previous pulses from PSP amplitudes. From the maximum membrane potential evoked by each pulse in an IPI train, we subtracted the minimum membrane potential within a window starting with the stimulus pulse offset and ending with the peak potential evoked by that pulse (see Materials and Methods). We found that temporal summation was critical for establishing high-pass responses (Fig. 8A, compare "observed" with "depression only" curves). Without summation, only 1 of 12 high-pass neurons (8\%) was classified as being high-pass. Five high-pass neurons (42\%) were classified as bandpass tuned, 3 (25\%) were classified as low-pass tuned, and $3(25 \%)$ were classified as complex tuned. Compared with high-pass neurons, removing the effects of summation in low-pass neurons had minimal effects on tuning (Fig. $8 B$, compare "observed" with "depression only" curves). The majority of low-pass neurons ( $n=17$ of 19 neurons; 89\%) remained lowpass tuned, with 1 neuron (5\%) becoming high-pass tuned and 1 neuron $(5 \%)$ becoming bandpass tuned. Without the effects of summation, tuning shifted toward low-pass and away from highpass (Fig. $8 C ; \chi_{(8)}^{2}=19, p<0.00095$ ).

Together, these results suggest that temporal summation and depression each contribute to establishing multiple tuning types, but that the greatest diversity of tuning occurs when summation and depression are both acting. Summation and depression are equally effective at producing low-pass responses, suggesting an inherent bias toward producing low-pass responses in the initial balance of excitation and inhibition as well as the degree of de-
A

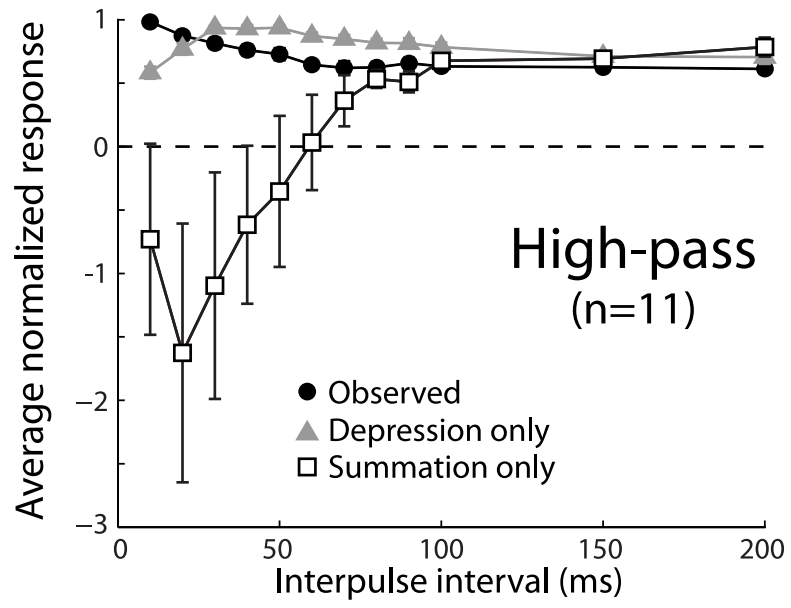

B
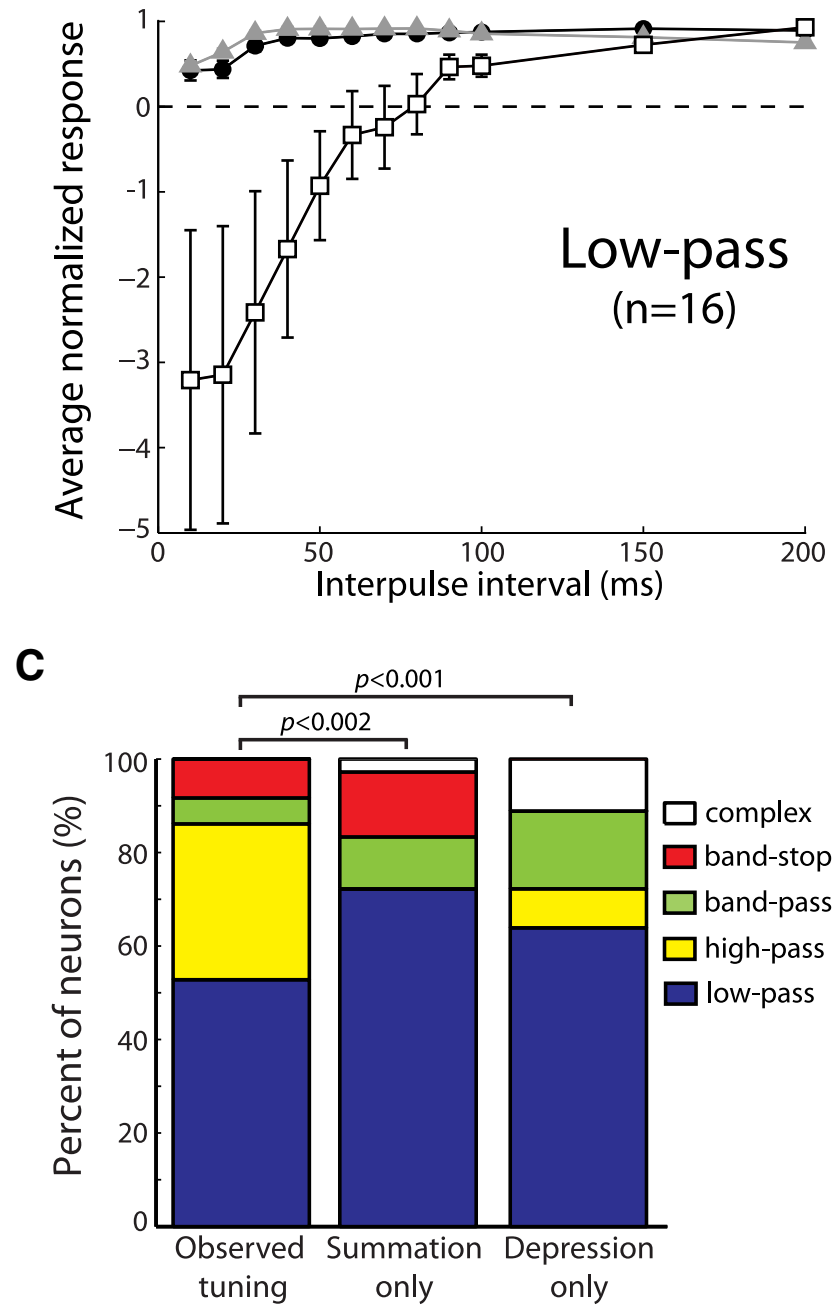

Figure 8. With temporal summation alone or depression alone, tuning among the population of ELp neurons would shift toward low-pass. $\boldsymbol{A}, \boldsymbol{B}$, Tuning curves of observed PSPs, PSPs after summation removal ("depression only"), and PSPs estimated from conductance convolutions ("summation only") in response to IPI stimuli from high-pass $(\boldsymbol{A})$ and low-pass $(\boldsymbol{B})$ neurons. Error bars represent SEM. $\boldsymbol{C}$, The percentage of neurons ( $n=36$ neurons) classified as each tuning type using the observed PSPs, the PSPs estimated from conductance convolutions, and the PSPs after summation removal. Reported $p$ values are the results of $\chi^{2}$ observed versus expected frequency tests. 

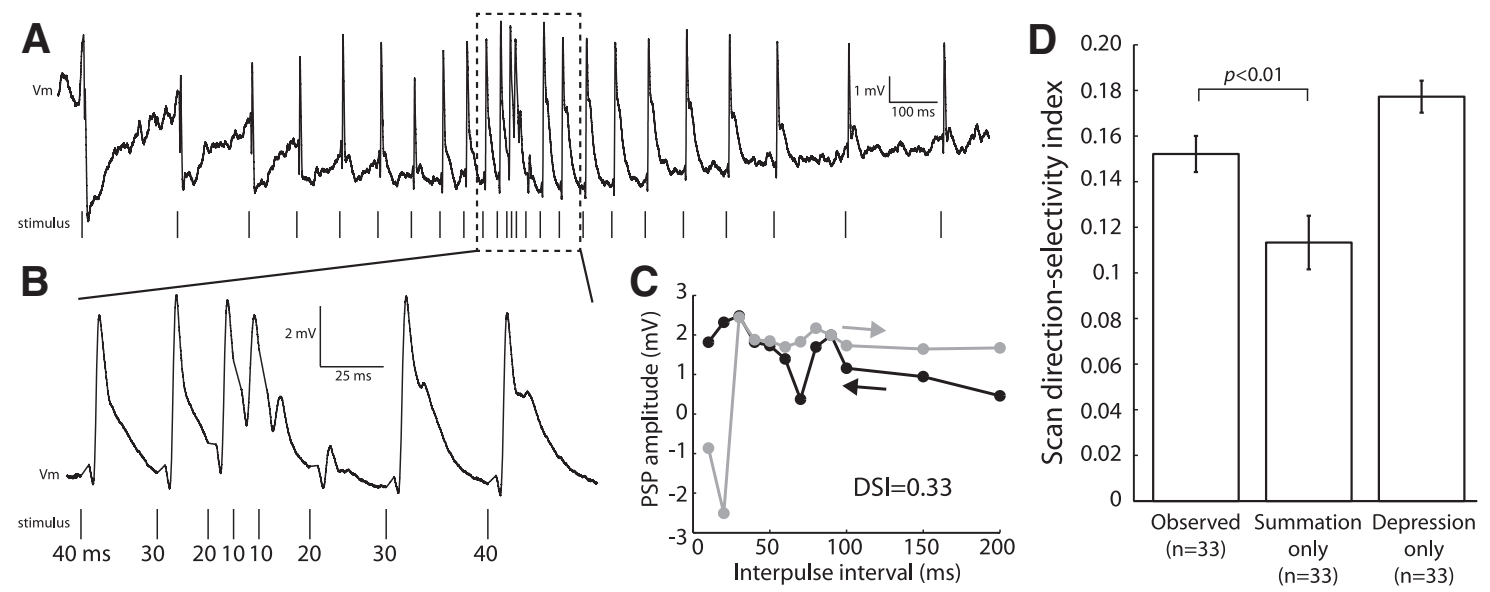

Figure 9. Depression increases selectivity for direction of interval change. $\boldsymbol{A}$, The $V_{m}$ values recorded from a low-pass neuron in response to a decreasing then an increasing IPI scan stimulus. $B$, Close-up of the responses to short intervals of the recording trace in $A$. Numbers below the stimulus tick marks indicate the IPI in milliseconds. $C, A$ plot of the PSP amplitudes of the neuron in $\boldsymbol{A}$ versus IPI for the decreasing (black) and increasing (gray) portions of the scan stimulus. The scan DSI for this neuron was 0.33 . D, Bar graph of the average scan DSI for observed PSPS, PSPs estimated from conductance convolutions ("summation only"), and PSPs after summation removal ("depression only"). Because there were no differences in DSI among high-pass, low-pass, bandpass, and bandstop neurons, neurons from all tuning groups were combined. The reported $p$ value is the result of a Tukey's post hoc test following a repeated-measures ANOVA.

pression of inputs. A combination of summation and depression is essential for establishing high-pass tuning and generating a greater diversity of IPI tuning among the population of ELp neurons.

Depression increases sensitivity to directional changes in IPI The responses of some ELp neurons are sensitive to the direction of interval change (Fig. 9A, B; Carlson, 2009). In the low-pass neuron shown in Figure 9, the amplitude of the PSPs evoked by the shortest and the longest intervals delivered depends upon the direction of interval change (Fig. 9C). To determine how depression and summation contribute to this directional sensitivity, we calculated a scan directional selectivity index (DSI) for recorded PSPs ("observed" in Fig. 9D), PSPs estimated from convolved conductances ("summation only" in Fig. 9D), and summationremoved PSPs ("depression only" in Fig. 9D) in response to IPI scans (see Materials and Methods). The scan DSI ranges from 0 (no direction selectivity) to 1 (maximum direction selectivity). ELp neurons exhibited a range of DSI values (range, 0.07-0.33), with no differences among the tuning groups (one-way ANOVA, $\left.F_{(3)}=0.56, p=0.64\right)$. Therefore, we combined high-pass, lowpass, bandpass, and bandstop neurons to investigate the effects of depression and summation. We limited our analysis to neurons in which the results of our summation removal method were linearly correlated with those of the true summation removal method at both 10 and 100 ms IPIs (see Materials and Methods).

Minimizing the effects of depression and summation significantly affected the scan DSI (repeated-measures ANOVA, $F_{(2)}=$ $13, p=0.00002)$. The DSI of the PSPs resulting from summation only ("without depression") was significantly smaller than that of the observed PSPs (Fig. 9D; Tukey's post hoc test, $p=0.0085$ ). This decrease in DSI was mostly due to the summating effects of inhibition that drove the estimated PSPs below the resting potentials of the neurons. In contrast, there was no difference between the DSIs of the observed PSPs and the PSPs resulting from depression only ("without summation"; Fig. 9D; Tukey's post hoc test, $p=0.12$ ). These results suggest that short-term depression contributes to the sensitivity of ELp neurons to changes in IPI.
Table 1. Mechanisms contributing to excitatory-inhibitory integration underlying high-pass and low-pass tuning

\begin{tabular}{lll}
\hline Mechanism & High-pass responses & Low-pass responses \\
\hline Short-term depression & More depression of inhibition & $\begin{array}{c}\text { More depression of excitation } \\
\text { (usually, but not always) }\end{array}$ \\
$\begin{array}{l}\text { Temporal summation } \\
\text { Onset duration }\end{array}$ & $\begin{array}{l}\text { More summation of excitation } \\
\text { Equal for excitation and } \\
\text { inhibition } \\
\text { Onset strength }\end{array}$ & $\begin{array}{c}\text { More summation of inhibition } \\
\text { Stronger inhibition }\end{array}$ \\
& & $\begin{array}{c}\text { Equal for excitation and } \\
\text { inhibition }\end{array}$ \\
\hline
\end{tabular}

\section{Discussion}

Our findings provide insight into the interactions between excitation and inhibition that contribute to behaviorally relevant interval tuning in vivo (Table 1). The strong responses of high-pass neurons at short intervals resulted from a combination of inhibition that depressed more than excitation, and temporal summation of excitation (Fig. 10B). By contrast, the weak responses of low-pass neurons at short intervals resulted from long-lasting inhibition, with an additional contribution from excitation that depressed more than inhibition in the majority of neurons (Fig. $10 C, D)$. Without short-term depression, low-pass tuning would dominate in ELp and neurons would be less sensitive to IPI sequences. On the other hand, without temporal summation, tuning would also shift toward low pass, but sensitivity to interval change would be unaffected. Therefore, the combination of short-term depression and temporal summation produces a more diverse range of interval tuning than either mechanism alone. Furthermore, depression in ELp increases the ability of the circuit to code for changing IPI sequences. Thus, both mechanisms working together enhance the ability of the circuit to detect communication signals, which are characterized both by their IPIs and by directional changes in IPIs (Carlson, 2002). Our results provide the first description of how different synaptic mechanisms can interact in vivo to establish neurons that code for behaviorally relevant stimulus intervals.

Low-pass neurons received inhibition that was longer than excitation, whereas high-pass neurons received inhibition and excitation of similar durations. The duration of conductance 

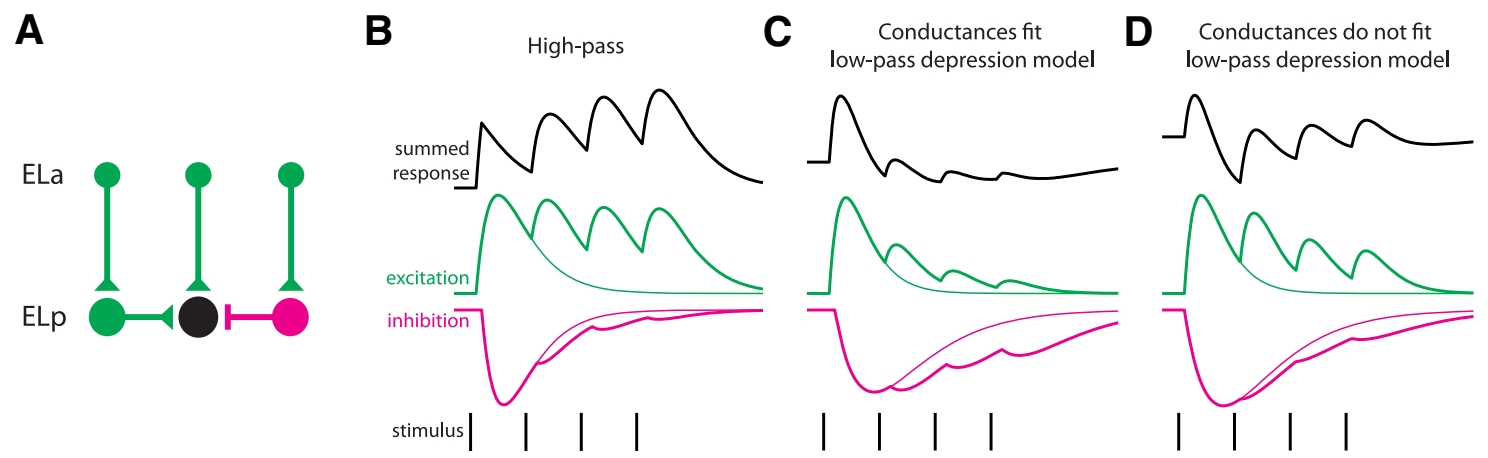

Figure 10. Schematic of ELp circuitry and synaptic mechanisms contributing to interval tuning. $A$, An ELp neuron (black) receives excitatory (green) inputs from ELa and from other ELp neurons, as well as inhibitory (magenta) inputs from other ELp neurons. $\boldsymbol{B}$, High-pass tuning is associated with excitation that depresses less than inhibition, leading to more temporal summation of excitation than inhibition. Excitation and inhibition elicited by the first stimulus pulse are indicated by thin lines. The summed response is the result of adding excitatory and inhibitory traces. $C$, The majority of low-pass neurons fit a depression model in which excitation depresses more than inhibition. Onset inhibition that lasts longer than onset excitation also contributes to low-pass responses. $\boldsymbol{D}$, A subset of low-pass neurons does not fit a depression model for low-pass tuning; instead, excitation depresses less than inhibition. Onset inhibition that lasts longer than onset excitation contributes to these neurons' low-pass responses.

changes can be affected not just by receptor/channel kinetics, but also by the relative timing and locations of synaptic inputs along the dendritic tree. Multiple, slightly asynchronous inhibitory inputs arriving at different locations on the postsynaptic membrane might give rise to weaker, but longer-lasting conductance changes than a few synchronous inputs arriving at the same location. Multiple studies have reported the effects of spatiotemporal activation of excitatory dendritic inputs on somatic potentials (Branco et al., 2010; Oviedo and Reyes, 2012; Abbas et al., 2013). Many additional parameters could affect the duration of conductance changes, including vesicle release dynamics; the distribution, density, and subunit composition of postsynaptic receptors; and ion channel kinetics (Euler and Denk, 2001; Farrant and Nusser, 2005).

Differences in the degrees of depression of excitation and inhibition also contributed to interval tuning. In addition to GABAergic inhibition from local interneurons (George et al., 2011), ELp neurons receive excitation from ELa small cells (Friedman and Hopkins, 1998; Lyons-Warren et al., 2013a) and from other ELp neurons (Ma et al., 2013; Fig. 10A). A feedback projection from the isthmic granule nucleus also terminates onto ELp neurons (Haugede-Carre, 1979); however, the nature of this input is unknown. Nevertheless, the large dendritic arborizations of ELp neurons and the relatively high probability of excitatory connections between pairs of ELp neurons suggest that they integrate a large number of inputs (Xu-Friedman and Hopkins, 1999; George et al., 2011; Ma et al., 2013). Recently, paired in vitro recordings revealed short-term depression at single excitatory connections between ELp neurons, with no differences in the amount of depression in relation to presynaptic or postsynaptic IPI tuning (Ma et al., 2013).

Therefore, three hypotheses for the origin of the observed differences in the depression of excitation and inhibition arise. First, since excitatory connections between ELp neurons depressed similarly regardless of IPI tuning (Ma et al., 2013), inhibitory synapses could depress to varying degrees onto different neurons. Differences in the depression of inhibition onto a presynaptic excitatory input could then create apparent differences in excitatory depression.

A second hypothesis is that all local ELp connections depress uniformly, but excitatory synapses from ELa depress with varying dynamics. Any variation in inhibitory depression would then reflect differences in the inputs from ELa onto inhibitory neurons.
Paired intracellular recordings of ELp neurons along with their inhibitory or ELa inputs would be necessary to characterize depression at these two types of synapses. Multiple studies have reported that the same output neuron can form synapses whose type and degree of short-term plasticity depends on the target cell (for review, see Blackman et al., 2013). The observed range of time courses and degrees of depression of both excitation and inhibition onto ELp neurons could result from diversity in the depression of ELa inputs as well as diversity in the depression of local inhibitory connections.

A third hypothesis for differences in the degree of depression of excitation and inhibition is based on local ELp network interactions. Excitatory connections are more likely to occur between ELp neurons of the same tuning class, and single excitatory connections between ELp neurons depress to similar degrees, regardless of presynaptic or postsynaptic IPI tuning (Ma et al., 2013). Since the spike output of high-pass neurons increases with shortinterval stimulation (Carlson, 2009), the convergence of many excitatory high-pass inputs onto a high-pass neuron could keep the excitatory conductance higher during repeated stimulation, even with depression. Furthermore, the majority of low-pass neurons received excitatory inputs that depressed more than inhibitory inputs at short intervals. Since the spike output of lowpass neurons decreases with short-interval stimulation (Carlson, 2009), the convergence of many excitatory low-pass inputs onto a low-pass ELp neuron could cause a rapid reduction in excitatory conductance during repetitive stimulation. Thus, the observed depression of synaptic conductances in ELp neurons may reflect the compounding effect of depression at many synapses within the local ELp circuitry.

ELp neurons exhibit large, spiny dendritic arbors that can span up to $200 \mu \mathrm{m}$ in diameter (Xu-Friedman and Hopkins, 1999; George et al., 2011). Passive electrical properties owing to morphology, as well as active dendritic conductances, can influence linear and nonlinear synaptic integration along dendrites (Magee, 2000; Gulledge et al., 2005; London and Häusser, 2005). We have not yet looked in detail for morphological differences between ELp neurons of different tuning types, but previous anatomical studies have not revealed any obvious morphological classes of ELp neurons (George et al., 2011; Ma et al., 2013). We did not find any differences in passive membrane properties with respect to IPI tuning in this study. Furthermore, we minimized possible contributions from active conductances by obtaining 
recordings in hyperpolarized current-clamp levels and by limiting our conductance estimates to portions of the responses in which the current-voltage relationship was linear. However, active dendritic processing could be another factor that might further shape IPI tuning beyond the synaptic mechanisms we described. Calcium imaging and glutamate- or GABA-uncaging experiments would be necessary to reveal possible contributions of active dendritic processing to IPI tuning.

Neurons sensitive to temporal patterns in sensory stimuli have been identified in the auditory midbrains of frogs (Alder and Rose, 1998) and bats (Grothe, 1994), cat auditory cortex (Sakai et al., 2009), and the electrosensory midbrain of wave-type weakly electric fish (Fortune and Rose, 1997a), which evolved an electrosensory system independently from the mormyrid fish that we studied. Interval-selective neurons in frog midbrains are tuned to the intervals between successive sound pulses (Alder and Rose, 1998), which vary across (but not within) distinct types of communication calls (Allen, 1973). Short-interval tuning in these neurons appears to arise from interactions between inhibition and enhancement of excitation, whereas long-interval tuning can arise from different combinations of mechanisms: the relative timing of excitation and inhibition, depression of excitation, or summation or enhancement of excitation, without a role for inhibition (Edwards et al., 2008; Rose et al., 2011). Neurons that are selective for slow beat rates $(2-8 \mathrm{~Hz})$ in wave-type electric fish midbrains get their temporal sensitivity through depression of excitation as well as passive electrical filtering and voltagedependent conductances (Rose and Fortune, 1999; Fortune and Rose, 2000). Whether inhibition also contributes to this tuning remains unknown.

In contrast to frog auditory and wave-type electric fish neurons, ELp neurons are involved in decoding intervals ranging from $\sim 10 \mathrm{~ms}$ up to hundreds of milliseconds (Hopkins, 1986). Mormyrid electric communication signals typically consist of a decrease then an increase in IPI, with distinct signals differing in the precise temporal sequence of IPIs (Carlson and Hopkins, 2004). Therefore, neural representations of the IPIs present in the wide range of signals used as well as the direction of IPI change is necessary for discriminating signals with different social functions. The combination of temporal summation and depression results in greater diversity of IPI tuning and sensitivity to the direction of IPI change than either mechanism alone. Detecting the direction of changing temporal patterns may not be as important for distinguishing frog communication signals or sinusoidal beat rates in wave-type electric fish, such that those circuits may be able to use fewer synaptic mechanisms to achieve the required temporal sensitivity.

Although excitatory-inhibitory interactions, short-term synaptic plasticity, and temporal summation have all been implicated in interval tuning, our study is the first to reveal how these multiple synaptic mechanisms interact in midbrain sensory neurons to establish behaviorally relevant interval tuning in vivo. It remains to be seen how variations in the balance of depression and summation in excitatory and inhibitory pathways arise in ELp neurons. Future studies of IPI tuning in ELp will seek to identify the contribution of network dynamics and local dendritic computations to this behaviorally relevant information processing.

\section{References}

Abbas SY, Hamade KC, Yang EJ, Nawy S, Smith RG, Pettit DL (2013) Directional summation in non-direction selective retinal ganglion cells. PLOS Comput Biol 9:e1002969. CrossRef Medline
Alder TB, Rose GJ (1998) Long-term temporal integration in the anuran auditory system. Nat Neurosci 1:519-523. CrossRef Medline

Allen DM (1973) Some relationships of vocalization to behavior in the pacific treefrog, Hyla regilla. Herpetologica 29:366-371.

Amagai S (1998) Time coding in the midbrain of mormyrid electric fish. II. Stimulus selectivity in the nucleus exterolateralis pars posterior. J Comp Physiol A Neuroethol Sens Neural Behav Physiol 182:131-143. CrossRef

Baker CA, Kohashi T, Lyons-Warren AM, Ma X, Carlson BA (2013) Multiplexed temporal coding of electiric communication signals in mormyrid fishes. J Exp Biol 216:2365-2379. CrossRef Medline

Bell CC, Grant K (1989) Corollary discharge inhibition and preservation of temporal information in a sensory nucleus of mormyrid electric fish. J Neurosci 9:1029-1044. Medline

Bennett MV (1965) Electroreceptors in mormyrids. Cold Spring Harb Symp Quant Biol 30:245-262. CrossRef Medline

Blackman AV, Abrahamsson T, Costa RP, Lalanne T, Sjöström PJ (2013) Target-cell-specific short-term plasticity in local circuits. Front Synaptic Neurosci 5:11. CrossRef Medline

Branco T, Clark BA, Häusser M (2010) Dendritic discrimination of temporal input sequences in cortical neurons. Science 329:1671-1675. CrossRef Medline

Carlson BA (2002) Electric signaling behavior and the mechanisms of electric organ discharge production in mormyrid fish. J Physiol Paris 96:405419. CrossRef Medline

Carlson BA (2009) Temporal-pattern recognition by single neurons in a sensory pathway devoted to social communication behavior. J Neurosci 29:9417-9428. CrossRef Medline

Carlson BA, Hopkins CD (2004) Stereotyped temporal patterns in electrical communication. Anim Behav 68:867-878. CrossRef

Carrasco A, Lomber SG (2011) Neuronal activation times to simple, complex, and natural sounds in cat primary and nonprimary auditory cortex. J Neurophysiol 106:1166-1178. CrossRef Medline

Edwards CJ, Alder TB, Rose GJ (2002) Auditory midbrain neurons that count. Nat Neurosci 5:934-936. CrossRef Medline

Edwards CJ, Leary CJ, Rose GJ (2008) Mechanisms of long-interval selectivity in midbrain auditory neurons: roles of excitation, inhibition, and plasticity. J Neurophysiol 100:3407-3416. CrossRef Medline

Euler T, Denk W (2001) Dendritic processing. Curr Opin Neurobiol 11: 415-422. CrossRef Medline

Farrant M, Nusser Z (2005) Variations on an inhibitory theme: phasic and tonic activation of GABA(A) receptors. Nat Rev Neurosci 6:215-229. CrossRef Medline

Fortune ES, Rose GJ (1997a) Temporal filtering properties of ampullary electrosensory neurons in the torus semicircularis of Eigenmannia: evolutionary and computational implications. Brain Behav Evol 49:312-323. CrossRef Medline

Fortune ES, Rose GJ (1997b) Passive and active membrane properties contribute to the temporal filtering properties of midbrain neurons in vivo. J Neurosci 17:3815-3825. Medline

Fortune ES, Rose GJ (2000) Short-term synaptic plasticity contributes to the temporal filtering of electrosensory information. J Neurosci 20:71227130. Medline

Friedman MA, Hopkins CD (1998) Neural substrates for species recognition in the time-coding electrosensory pathway of mormyrid electric fish. J Neurosci 18:1171-1185. Medline

Geis HR, Borst JG (2013) Intracellular responses to frequency modulated tones in the dorsal cortex of the mouse inferior colliculus. Front Neural Circuits 7:7. CrossRef Medline

George AA, Lyons-Warren AM, Ma X, Carlson BA (2011) A diversity of synaptic filters are created by temporal summation of excitation and inhibition. J Neurosci 31:14721-14734. CrossRef Medline

Gittelman JX, Li N, Pollak GD (2009) Mechanisms underlying directional selectivity for frequency-modulated sweeps in the inferior colliculus revealed by in vivo whole-cell recordings. J Neurosci 29:13030-13041. CrossRef Medline

Goel A, Buonomano DV (2014) Timing as an intrinsic property of neural networks: evidence from in vivo and in vitro experiments. Philos Trans R Soc Lond B Biol Sci 369:20120460. CrossRef Medline

Groh JM, Kelly KA, Underhill AM (2003) A monotonic code for sound azimuth in primate inferior colliculus. J Cogn Neurosci 15:1217-1231. CrossRef Medline

Grothe B (1994) Interaction of excitation and inhibition in processing of 
pure tone and amplitude-modulated stimuli in the medial superior olive of the mustached bat. J Neurophysiol 71:706-721. Medline

Guillamon A, McLaughlin DW, Rinzel J (2006) Estimation of synaptic conductances. J Physiol Paris 100:31-42. CrossRef Medline

Gulledge AT, Kampa BM, Stuart GJ (2005) Synaptic integration in dendritic trees. J Neurobiol 64:75-90. CrossRef Medline

Haugede-Carre F (1979) Mesencephalic exterolateral posterior nucleus of the mormyrid fish Bryenomyrus niger-efferent connections studied by the HRP method. Brain Res 178:179-184. CrossRef Medline

Hedwig B, Knepper M (1992) Separation of synaptic and spike activity in intracellular recordings for selective analysis. J Neurosci Methods 42:8390. CrossRef Medline

Higley MJ, Contreras D (2006) Balanced excitation and inhibition determine spike timing during frequency adaptation. J Neurosci 26:448-457. CrossRef Medline

Hopkins CD (1986) Behavior of mormyridai. In: Electroreception (Bullock TH, Heiligenberg W, eds), pp 527-576. New York: Wiley.

Klyachko VA, Stevens CF (2006) Excitatory and feed-forward inhibitory hippocampal synapses work synergistically as an adaptive filter of natural spike trains. PLoS Biol 4:e207. CrossRef Medline

Kohashi T, Carlson BA (2014) A fast BK-type KCa current acts as a postsynaptic modulator of temporal selectivity for communication signals. Front Cell Neurosci 8:286. CrossRef

London M, Häusser M (2005) Dendritic computation. Annu Rev Neurosci 28:503-532. CrossRef Medline

Lyons-Warren AM, Kohashi T, Mennerick S, Carlson BA (2013a) Detection of submillisecond spike timing differences based on delay-line anticoincidence detection. J Neurophysiol 110:2295-2311. CrossRef Medline

Lyons-Warren AM, Kohashi T, Mennerick S, Carlson BA (2013b) Retrograde fluorescent labeling allows for targeted extracellular single-unit recording from identified neurons in vivo. J Vis Exp. Advance online publication. Retrieved September 11, 2014. doi:10.3791/3921. CrossRef Medline

Ma X, Kohashi T, Carlson BA (2013) Extensive excitatory network interactions shape temporal processing of communication signals in a model sensory system. J Neurophysiol 110:456-469. CrossRef Medline

Magee JC (2000) Dendritic integration of excitatory synaptic input. Nat Rev Neurosci 1:181-190. CrossRef Medline
Monier C, Fournier J, Frégnac Y (2008) In vitro and in vivo measures of evoked excitatory and inhibitory conductance dynamics in sensory cortices. J Neurosci Methods 169:323-365. CrossRef Medline

Oviedo HV, Reyes AD (2012) Integration of subthreshold and suprathreshold excitatory barrages along the somatodendritic axis of pyramidal neurons. PLoS One 7:e33831. CrossRef Medline

Pluta SR, Kawasaki M (2010) Temporal selectivity in midbrain electrosensory neurons identified by modal variation in active sensing. J Neurophysiol 104:498-507. CrossRef Medline

Priebe NJ, Ferster D (2005) Direction selectivity of excitation and inhibition in simple cells of the cat primary visual cortex. Neuron 45:133-145. CrossRef Medline

Rose G, Capranica RR (1983) Temporal selectivity in the central auditory system of the leopard frog. Science 219:1087-1089. CrossRef Medline

Rose GJ, Fortune ES (1996) New techniques for making whole-cell recordings from CNS neurons in vivo. Neurosci Res 26:89-94. Medline

Rose GJ, Fortune ES (1999) Mechanisms for generating temporal filters in the electrosensory system. J Exp Biol 202:1281-1289. Medline

Rose GJ, Leary CJ, Edwards CJ (2011) Interval-counting neurons in the anuran auditory midbrain: factors underlying diversity of interval tuning. J Comp Physiol A Neuroethol Sens Neural Behav Physiol 197:97-108. CrossRef Medline

Sakai M, Chimoto S, Qin L, Sato Y (2009) Neural mechanisms of interstimulus interval-dependent responses in the primary auditory cortex of awake cats. BMC Neurosci 10:10. CrossRef Medline

Wehr M, Zador AM (2003) Balanced inhibition underlies tuning and sharpens spike timing in auditory cortex. Nature 426:442-446. CrossRef Medline

Williams AJ, Fuzessery ZM (2012) Multiple mechanisms shape FM sweep rate selectivity: complementary or redundant? Front Neural Circuits 6:54. CrossRef Medline

Wong RY, Hopkins CD (2007) Electrical and behavioral courtship displays in the mormyrid fish Brienomyrus brachyistius. J Exp Biol 210:22442252. CrossRef Medline

Xu-Friedman MA, Hopkins CD (1999) Central mechanisms of temporal analysis in the knollenorgan pathway of mormyrid electric fish. J Exp Biol 202:1311-1318. Medline 